\title{
Detailed performance characteristics of vertically polarized, cylindrical, active coated nano-particle antennas
}

\author{
Junping Geng, ${ }^{1}$ Richard W. Ziolkowski, ${ }^{2}$ Ronghong Jin, ${ }^{1}$ and Xianling Liang ${ }^{1}$ \\ Received 15 October 2011; revised 5 January 2012; accepted 21 February 2012; published 12 April 2012.
}

[1] The electromagnetic properties of active cylindrical coated nano-particle antennas are investigated. It is demonstrated that the active cylindrical coated nano-particle, whether illuminated by a plane wave or an electric Hertzian dipole (EHD) (small current) element, acts as a strong dipole radiator at its resonant frequency. It is shown that the plane wave scattering cross section could be increased by about $40 \mathrm{dBsm}$, and the maximum peak of the power radiated by an EHD element could be increased more than $65 \mathrm{~dB}$ in the presence of the active nano-particle over its value when radiating into free space. An array, constructed with four active cylindrical coated nano-particles and excited by an EHD element located at or near its center is also studied. Large directivity values, more than $8 \mathrm{~dB}$, are obtained for particular array configurations and EHD locations.

Citation: Geng, J., R. W. Ziolkowski, R. Jin, and X. Liang (2012), Detailed performance characteristics of vertically polarized, cylindrical, active coated nano-particle antennas, Radio Sci., 47, RS2013, doi:10.1029/2011RS004898.

\section{Introduction}

[2] Nano-particles and nano-structures have been shown to have attractive prospects for advancements in many areas, including, for example, biology and medicine [Hirsch et al., 2006; Jain et al., 2008; Liu and Huo, 2009], efficient solar cells [Catchpole and Polman, 2008; Ferry et al., 2008; Pala et al., 2009; Atwater and Polman, 2010], and subwavelength resolution imaging [Atwater and Polman, 2010; Shvets et al., 2007; Grbic et al., 2008; Kawata et al., 2009]. They have been considered as potential nano-sensors that could provide non-invasive measurements of the electromagnetic properties of molecules or other nano-particles [Lin et al., 2005; Gordon and Ziolkowski, 2007a; Halas, 2010]. Because of the resulting surface plasmonic characteristics realized by combining metals at optical frequencies, which acquire epsilon negative (ENG) properties, with normal dielectrics, which have double positive (DPS) properties, one can design resonant nano-sized plasmonic particles that could be used to achieve highly sub-wavelength lasing elements and nanoamplifiers [Gordon and Ziolkowski, 2007a; Stockman, 2010; Arslanagić and Ziolkowski, 2010]. Highly subwavelength spherical core-shell plasmonic nano-particles, i.e., coated nano-particles (CNP) which consist of spherical nano-shells surrounding spherical cores, have been studied in all their metal-dielectric combinations. They can be designed to have novel optical properties. These include resonant scattering and transparency effects, which exhibit geometric tunability. If the materials of the CNP are lossless or lossy, the system is

\footnotetext{
${ }^{1}$ Electronic Engineering Department, Shanghai Jiao Tong University, Shanghai, China.

${ }^{2}$ Department of Electrical and Computer Engineering, University of Arizona, Tucson, Arizona, USA.

Copyright 2012 by the American Geophysical Union. 0048-6604/12/2011RS004898
}

called a passive CNP, the model of which has discussed completely by Aden and Kerker [1951] and Arslanagic et al. [2007]. On the other hand, if gain material is included in the core or shell, it is called an active CNP. A spherical active CNP has been designed and tested to demonstrate a spaserbased nanolaser [Noginov et al., 2009].

[3] Losses remain one of the main disadvantages if metals are used in the optical frequency designs. They significantly constrain the possible enhancements of the resonant passive CNPs. Subwavelength silver CNPs can be tunable over the visible part of the spectrum. Consequently, gain material has been introduced to overcome those losses and to increase the enhancements. Silver is less lossy than gold over the visible part of the spectrum and will be emphasized in the designs reported below.

[4] Many of the present nano-antenna models involve canonical structures, e.g., spherical nano-particles. The canonical models are well-suited to analytical or semianalytical methods to characterize their performance. Consequently, these analytical approaches have been emphasized in numerous initial studies [e.g. Arslanagic et al., 2007; Arslanagic and Ziolkowski, 2010]. However, while they provide physical understanding, many canonical structures are far from being realizable with existing nano-fabrication technologies. Moreover, much more complex coated structures [e.g., Khlebtsov et al., 2011], are now being considered as those nano-fab techniques become more sophisticated and increased functionality is desired. These more complicated nano-structures, which have far more complex material and structural characteristics, can be modeled accurately only with proven numerical simulators.

[5] The numerical studies of one such nano-antenna geometry, a finite, closed, cylindrical coated nano-particle (CC-CNP), are reported in this paper. The selected geometry is a nano-structure version of a successful metamaterialbased UHF antenna [Erentok et al., 2009]. The CC-CNPs 
are essentially pillar nano-structures, which are now readily fabricated [e.g., Hill, 2010; Li et al., 2011]. A cylindrical gain impregnated silica core is surrounded by a silver coating and is excited with both a plane wave and an electric Hertzian dipole (EHD) element. It will be demonstrated that both the passive and active CC-CNP configurations resonantly produce the basic dipole mode and, hence, have a strong linear polarization characteristic. Parameter studies of the design will be used to illustrate various optical properties of these nano-antennas, especially their enhancements of the total radiated power. Results for a related open version have also shown very similar, interesting performance characteristics [Geng et al., 2011a]. The fully encapsulated gainimpregnated core is emphasized here because of its potential usefulness as an individual nano-amplifier. Finally, because of recent interest in controlling the directivity of the fields radiated by nano-antenna structures [Li et al., 2007; Mirin et al., 2010; Wang et al., 2011], the behavior of an array of CC-CNPs is also presented. The use of arrays of radiating elements to achieve high directivity patterns has a long history in the microwave domain. As will be shown with a nano-array consisting of four active CC-CNPs and excited by an EHD, similar significant enhancements of the directivity of the emitted far-fields can be achieve from particular designs.

\section{Numerical Models}

[6] The CC-CNP design problems were analyzed with CST Microwave Studio. It is a computational electromagnetic simulation tool set which solves Maxwell's equations in the time domain based on the Finite Integration in Time (FIT) technique (a slight generalization of the Finite Difference Time Domain (FDTD) method), and in the frequency domain with the Finite Element Method (FEM) [Weiland, 1998]. For wide band EM problems, the time domain methods are a good choice. On the other hand, frequency domain methods are generally more appropriate for narrow band problems. In addition to providing both time and frequency domain simulation capabilities, the CST software also has a user friendly interface and a powerful post processing environment that allows one to tailor the output results. It has been validated against experimental results for a wide variety of electromagnetic applications.

[7] With any lossy real or artificial material, dispersive effects must be taken into account. Details of the passive model used for silver, which accounts for the sub-100 nm thickness of the shell and interband transitions wavelengths between $200 \mathrm{~nm}$ to $1800 \mathrm{~nm}$, as well as recovers known empirical values, have been reported elsewhere [Johnson and Christy, 1972]. The gain model, as implemented in the frequency domain solver in CST, was described in detail by Geng et al. [2011b]. In particular, the permittivity is decomposed into a size dependent Drude response and an interband transition response as $\varepsilon(\omega, R)=\varepsilon_{\text {Drude }}(\omega, R)+$ $\chi_{\text {Interband }}(\omega)$, where the term $R$ taken to be the thickness of the metal shell and the Drude relative permittivity is given by the expression,

$$
\varepsilon_{\text {Drude }}(\omega, R)=1-\frac{\omega_{p}^{2}}{\Gamma(R)^{2}+\omega^{2}}+j \frac{\Gamma(R)^{2} \omega_{p}^{2}}{\omega\left(\Gamma(R)^{2}+\omega^{2}\right)}
$$

where $\omega_{\mathrm{p}}$ and $\Gamma$ are, respectively, the plasma and the collision frequencies. The damping frequency is assumed to take the form $\Gamma(R)=\Gamma_{\infty}+A V_{F} / R$ where $R$ is taken to be the thickness of the shell, $\mathrm{A}$ is a constant term assumed to be unity, i.e., $A=1.0$, and the term $V_{F}$ is the Fermi velocity. The parameters used for silver in our simulations were: $\mathrm{V}_{\mathrm{F}}=$ $1.39 \times 10^{6} \mathrm{~m} / \mathrm{s}$; the effective and actual mass ratio of the electron, $\mathrm{m}^{*} / \mathrm{m}=0.96$; and the number density of electrons, $\mathrm{N}_{\mathrm{e}}=5.85 \times 10^{28} \mathrm{~m}^{-3}$. The latter terms give the plasma frequency $\omega_{\mathrm{p}}=\sqrt{N_{e} e^{2} / m^{*} \varepsilon_{0}}=1.39269 \times 10^{13} \mathrm{~s}^{-1}$.

[8] The cores of the passive models of the CNPs were filled with silicon dioxide, $\mathrm{SiO}_{2}$. Because $\mathrm{SiO}_{2}$ has very low loss characteristics, we can set the relative epsilon to be a constant $\varepsilon_{\mathrm{r}}=2.05$, yielding the refractive index $n=\sqrt{\varepsilon_{r}}=$ 1.432. However, when losses are present, the refractive index expression contains an imaginary term, $\kappa$. The relative permittivity and the real and imaginary parts of the refractive index are then related by the expression:

$$
\varepsilon_{r}=(n-j \kappa)^{2}=n^{2}-\kappa^{2}-j 2 n \kappa
$$

where $\kappa$ is varied over a range of values that represent the expected loss or gain in the core of the nano-shell. In particular, $\kappa>0,=0$, and $<0$, respectively, for a lossy, lossless, and gain medium. It was assumed that the core was impregnated with atoms that were pumped into an excited state and which decayed to a lower energy level transferring energy to the field in the core produced by the incident plane wave (probe field) interacting with the core-shell system. This amplification process can be described by a steady state three- or four-level atom model. The resulting gain medium in the core can then be described by a canonical gain model, i.e., by values of the relative permittivity of the core: $\varepsilon_{r}$, as a function of the frequency. In particular, to incorporate a canonical gain model into CST Microwave Studio, the Lorentz model for the effective relative permittivity in the core:

$$
\varepsilon_{r}(\omega)=\varepsilon_{\infty}+\frac{\left(\varepsilon_{S}-\varepsilon_{\infty}\right) \omega_{0}^{2}}{\omega_{0}^{2}+j \omega \Gamma-\omega^{2}}
$$

is used. To recover the value $\kappa=-0.25$, which has been found optimal for related geometries at excitation frequencies around $600 \mathrm{THz}(500 \mathrm{~nm}$ wavelength) [e.g., Gordon and Ziolkowski, 2007a; Arslanagić and Ziolkowski, 2010; Gordon and Ziolkowski, 2007b], we set $f=f_{0}=600 \mathrm{THz}$ and $\omega=\omega_{0}=2 \pi f_{0}$, to obtain

$$
\varepsilon_{r}\left(\omega_{0}=2 \pi f_{0}\right)=\varepsilon_{\infty}+\frac{\left(\varepsilon_{S}-\varepsilon_{\infty}\right) \omega_{0}}{j \Gamma} .
$$

[9] This relation is then used to calculate $\varepsilon_{\infty}=1.9875$ and $\left(\varepsilon_{S}-\varepsilon_{\infty}\right) \omega_{0} / \Gamma=2 n \kappa$, so that

$$
\varepsilon_{S}=\frac{2 n \kappa \Gamma}{\omega_{0}}+\varepsilon_{\infty}=\frac{2 n \kappa \Gamma}{\omega_{0}}+1.9875 .
$$

[10] The collision frequency was set to the lower loss value [Geng et al., 2011b]: $\Gamma=10^{-3} \omega_{0}$.

[11] For a plane wave excitation of the CC-CNP, the behavior of the resulting scattered fields can be described by the scattering and absorption cross sections. The scattering cross-section is defined as the total integrated power 

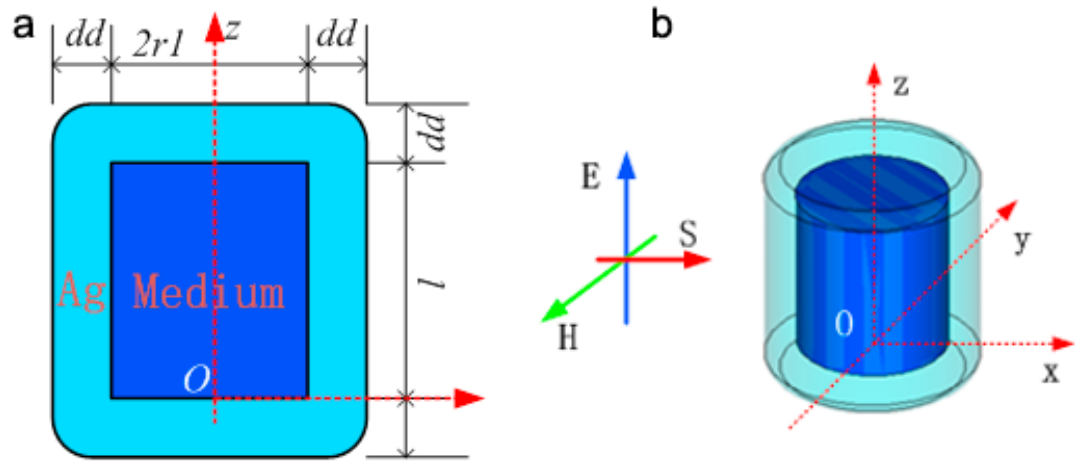

Figure 1. CC-CNP model with a plane wave excitation. (a) Structure and parameters and (b) vertically polarized plane wave: $\vec{E}, \vec{H}$ and $\vec{S}$ are oriented, respectively, along the $\mathrm{z}, \mathrm{y}$, and $\mathrm{x}$ axes.

contained in the scattered field normalized by the irradiance of the incident field and the absorption cross-section is defined by the net flux through a surface surrounding the concentric shells normalized by the incident field irradiance, and is thus a measure of how much energy is absorbed by the concentric shell structure. The scattering and absorption cross-sections can be expressed via Poynting's theorem through the scattered and absorbed powers, which are given, respectively, by the following expressions:

$$
\begin{gathered}
P_{s c a t}=\operatorname{Re}\left\{\frac{1}{2} \oiint_{S}\left[\vec{E}_{S} \times \vec{H}_{S}^{*}\right] \cdot \hat{n} d S\right\} \\
P_{a b s}=-\operatorname{Re}\left\{\frac{1}{2} \oiint_{S}\left[\vec{E}_{t o t} \times \vec{H}_{t o t}^{*}\right] \cdot \hat{n} d S\right\}
\end{gathered}
$$

where $S$ is a surface that surrounds the CC-CNP and is the unit outward pointing normal to that surface. Note that the incident, scattered, and total fields will be labeled by the subscripts inc, scat, and tot, respectively. These quantities are readily calculated using a cube as the surface. The total scattering cross-section, absorption cross-section and extinction cross-section are then defined from the ratio of the scattered or absorbed power to the incident irradiance $I_{\text {inc }}$, as: $\sigma_{\text {scat }}=P_{\text {scat }} / I_{\text {inc }}, \sigma_{a b s}=P_{a b s} / I_{\text {inc }}$ and $\sigma_{\text {ext }}=\sigma_{\text {scat }}+\sigma_{a b s}$.

[12] In a similar manner, for an EHD excitation of the CC-CNP, the response can be characterized by the total radiated power:

$$
P_{t o t}=\lim _{r \rightarrow \infty} \frac{1}{2} \int_{\theta=0}^{\pi} \int_{\varphi=0}^{2 \pi} \operatorname{Re}\left\{\vec{E}_{t o t}(r, \theta, \varphi) \times \vec{H}_{t o t}^{*}(r, \theta, \varphi)\right\} \cdot d \vec{S} .
$$

[13] Again, this quantity is readily calculated using a cube surrounding the EHD and CC-CNP system. The power radiated by an EHD, which is represented by the current element $p_{s}=I_{0} d$, alone in free space has the well-known value [Balanis, 2005]

$$
P_{E H D}=\frac{\eta_{0} \pi}{3}\left|\frac{p_{s} k_{0}}{2 \pi}\right|^{2}=\frac{\eta_{0} \pi}{3}\left|\frac{I_{0} d}{\lambda}\right|^{2}
$$

where $\eta_{0}$ and $\mathrm{k}_{0}$ are, respectively, the free space wave impedance and wave number. The behavior of the EHD-CCCNP nano-antenna will then be described by the power ratio: $P R=P_{t o t} / P_{E H D}$.
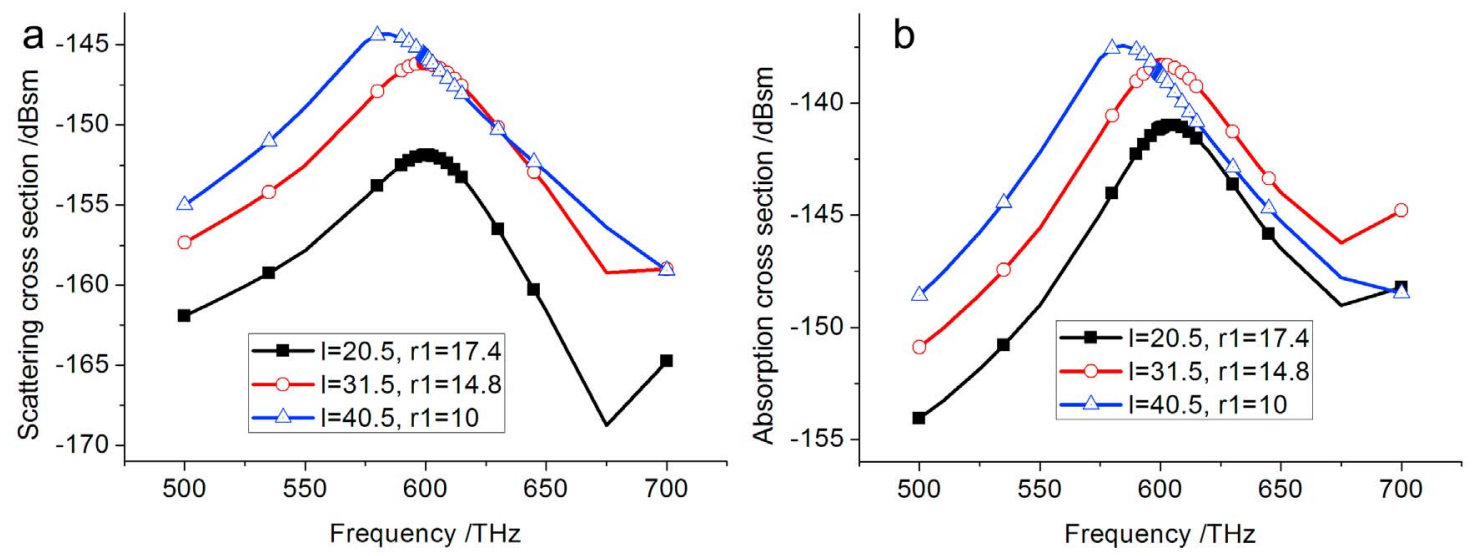

Figure 2. Excitation of the passive CC-CNP by a vertically polarized plane wave, sweeping the dimensions from $r 1: 10.0 \rightarrow 17.4 \mathrm{~nm}$ and $l: 23.5 \rightarrow 43.5 \mathrm{~nm}$. (a) Scattering and (b) absorption cross section values for the indicated cases. 


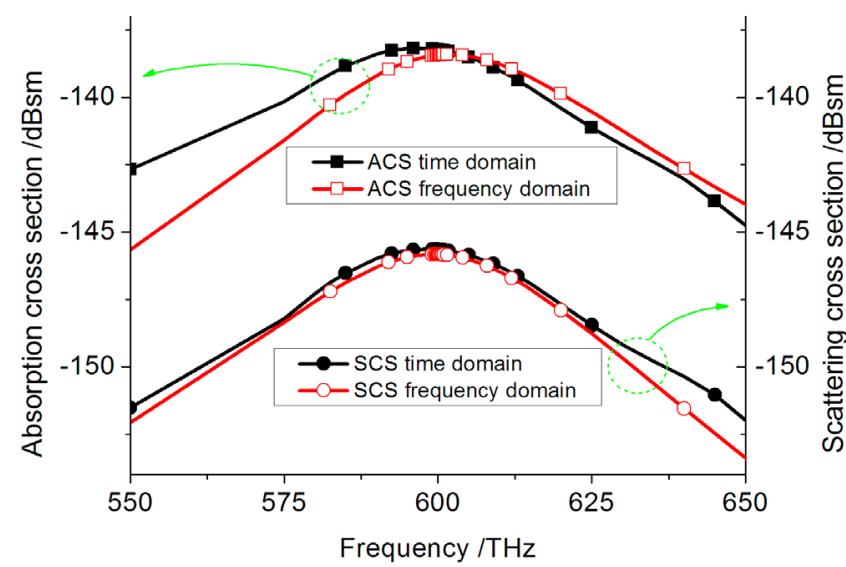

Figure 3. Scattering and absorption cross section curves predicted for the passive CC-CNP excited by the vertically polarized plane wave by the CST frequency and time domain solvers when $r 1=14.8 \mathrm{~nm}, l=34.5 \mathrm{~nm}$ and $d d=6.0 \mathrm{~nm}$.
[14] The performance characteristics expressed by equations (6)-(9) are calculated with the post-processing tools provided by the CST software. In particular, by implementing these formulas in the post processing environment, one obtains the desired values.

\section{Passive CC-CNP Numerical Results}

[15] The closed cylindrical coated nano-particle (CC-CNP) model and the coordinate system used in the CST model are shown in Figure 1. The thickness of the silver layer is $d d$. The inner cavity has radius $r 1$ and height $l$. Unlike spherical CNPs, whose response is polarization independent, the CCCNP has different responses for vertical (parallel to the particle axis) and horizontal (perpendicular to the particle axis) polarized excitations. The excitation of the CC-CNP by a vertically polarized plane wave propagating along the $\mathrm{z}$ axis, the axis of the cylinder, is shown in Figure $1 \mathrm{~b}$. The incident plane wavefield is explicitly given as:

$$
\vec{E}=\hat{z} E_{0} \cos (\omega t-k x)
$$

a

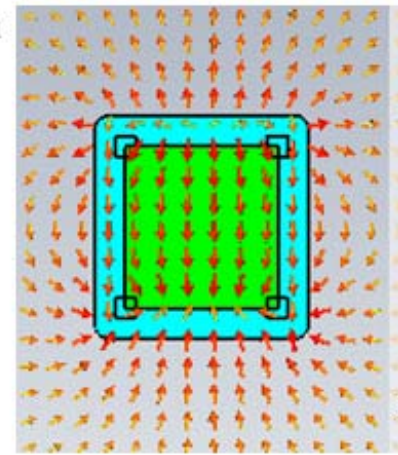

C

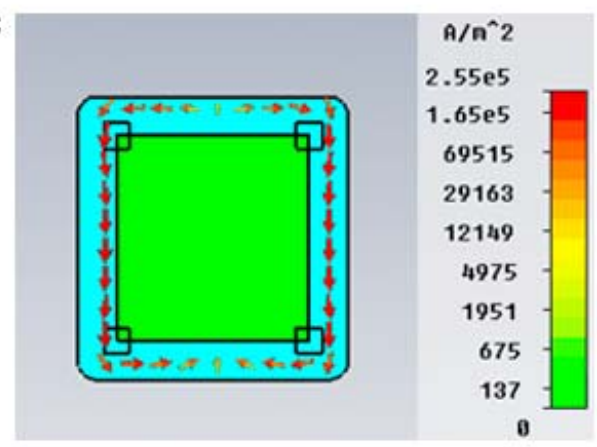

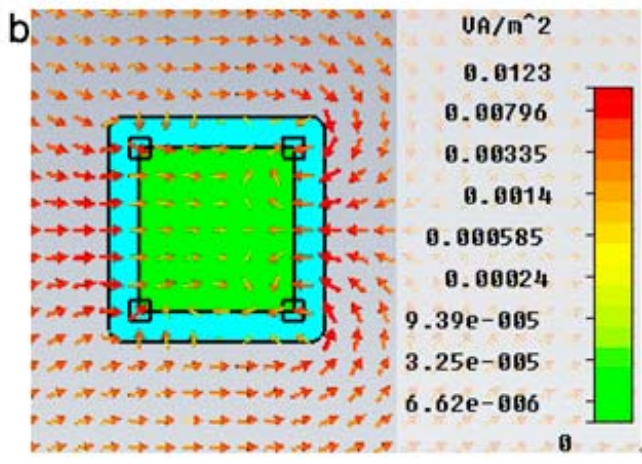

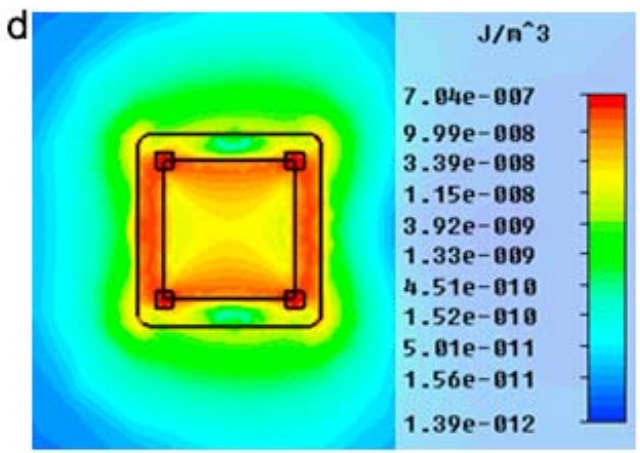

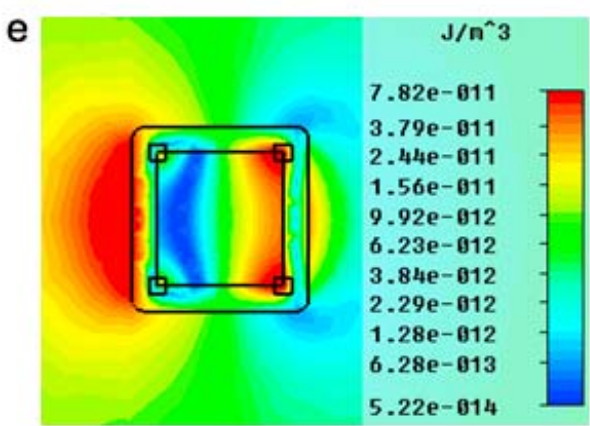

Figure 4. Field distributions in the y-plane at $\mathrm{f}=600 \mathrm{THz}$ for the $r 1=14.8 \mathrm{~nm}$ and $l=31.5 \mathrm{~nm}$ case: (a) E field, (b) power flow, (c) current density, (d) E-energy density, and (e) H-energy density. 

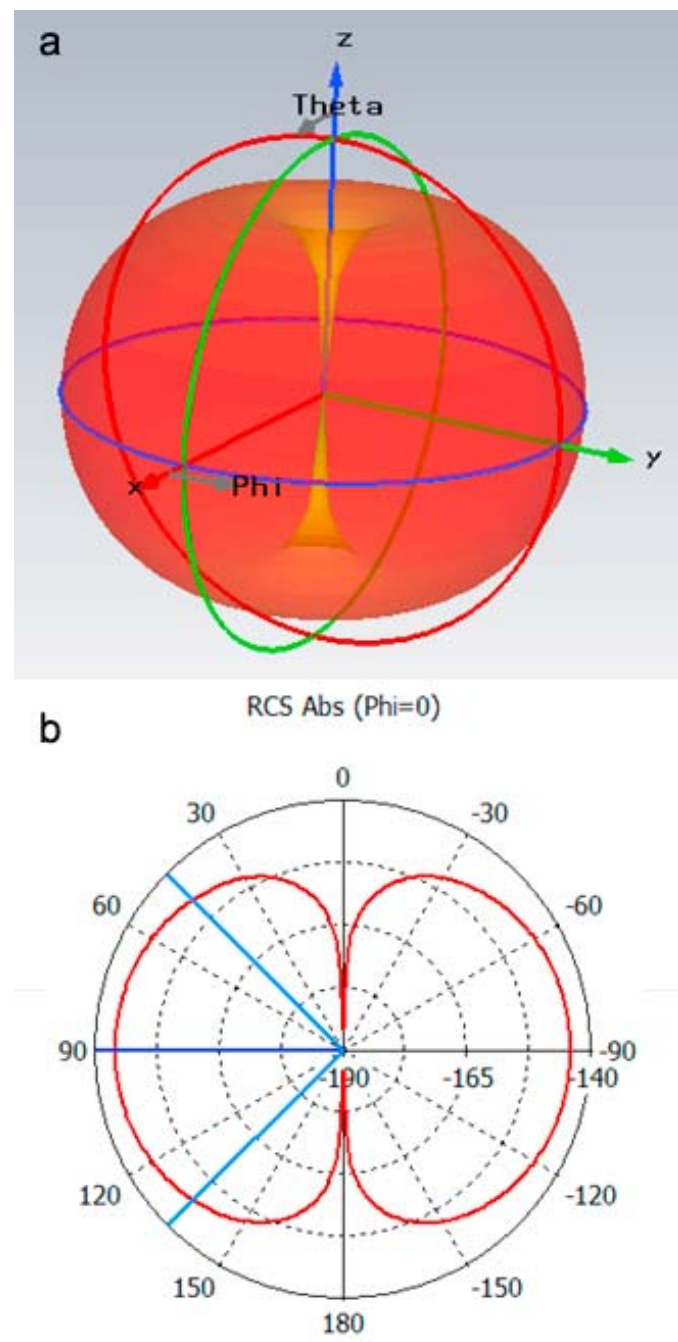

Theta / Degree vs. dBsm

Figure 5. The far-field behavior at the $600 \mathrm{THz}$ resonance frequency of the passive CC-CNP; (a) 3D radar cross-section (RCS) and (b) 2D cut of the RCS in the $\phi=0^{\circ}$ plane.

where $\mathrm{E}_{0}$ is assumed to be $1.0 \mathrm{~V} / \mathrm{m}$ throughout. Based on previous results for passive and active spherical CNPs, the thickness of the silver shell is set at $d d=6.0 \mathrm{~nm}$, Sweeping the parameters $r 1$ and $l$, the scattering cross section (SCS) and absorption cross section (ACS) for the passive CC-CNP are shown in Figure 2. There is a clear resonance peak for the scattering cross sections near $\mathrm{f}=600 \mathrm{THz}$ in Figure 2a. The peak location varies slowly as the parameters $r 1$ and $l$ are varied. The $3 \mathrm{~dB}$ bandwidth is about $50 \mathrm{THz}$. For $r 1=$ $14.8 \mathrm{~nm}$ and $l=31.5 \mathrm{~nm}$, the SCS peak (maximum) and ACS maximum occur at $600 \mathrm{THz}$.

[16] While the CST frequency domain solver can be used to simulate the behaviors of active CC-CNPs [Geng et al., 2011b], the time domain solver cannot [Geng et al., 2011b]. On the other hand, the time domain solver can model this passive CC-CNP. Consequently, to verify the frequency domain results with an independent computational electromagnetics approach, the passive CC-CNP cross-section results were also obtained with the time domain solver. These are compared in Figure 3 for the $r 1=$ $14.8 \mathrm{~nm}$ and $l=31.5 \mathrm{~nm}$ case, which is resonant at $600 \mathrm{THz}$. The trends of the cross-section curves from these two methods are very similar; the predicted resonant peaks are very close. As verified with simpler geometries, the slight discrepancies are caused by the coarser time domain grid.

[17] The frequency domain predicted distributions and vector fields for the electric field, the power density, current density and the electric and magnetic field energy densities of the passive CC-CNP at $600 \mathrm{THz}$ for the same case are shown in Figure 4. The total electric field distribution shown in Figure 4a is nearly symmetric despite the incident field being included, indicating that the scattered field generated by the resonant CC-CNP dominates the near-field behavior. It has a peak value $\left|\mathrm{E}_{\max }\right|=10.8818 \mathrm{~V} / \mathrm{m}$, a more than 10 times enhancement of the incident field. The power flow distribution in Figure $4 \mathrm{~b}$ shows how the CC-CNP has a SCS much larger than its geometrical size. Power is being channeled into the center of the CC-CNP from all sides at the resonance frequency. As shown in Figure 4c, the bulk currents at that resonance frequency are flowing strongly in the silver shell from its North pole to its South pole, a pattern which radiates the electric dipole mode, i.e., the fundamental TM dipole mode of this structure with the dipole being oriented in the same direction as the incident electric field, and explains the electric field distribution in Figure 4a. The same dipole mode is illustrated in Figures $4 \mathrm{~d}$ and $4 \mathrm{e}$. The electric energy density is mostly uniform throughout the core with peaks near the metallic corners where field singularities exist. The magnetic field energy density clearly has a dipole distribution. The generation of the dipole mode at the resonance frequency is further confirmed with the radar cross section plots given in Figure 5.

\section{Active CC-CNP Numerical Results: Plane Wave Excitation}

[18] The active CC-CNP has a gain material-doped $\mathrm{SiO}_{2}$ cylindrical core. The shell thickness is the same as those for the resonant passive case. The source is first taken to be the plane wave (1). Thus this configuration is geometrically the same as the one shown in Figure 1. Sweeping the height $l$ and the radius $r 1$ of the core in the active CC-CNP to find its maximum response, one obtains the cross-section results given in Figure 6.

[19] These sweeps indicate that the response is maximized with the parameter values $l=31.5 \mathrm{~nm}$ and $r 1=15.5 \mathrm{~nm}$ for $d d=6.0 \mathrm{~nm}$. The resonance frequency for these values is $600.05 \mathrm{THz}$. The slight change from the passive case is due to the impact of the gain material on the dielectric constant of the core. Comparing these results for this optimized active case with those given in Figure 3 for the passive case, it is very clear that at its resonance frequency the active CC-CNP significantly increases the SCS by about $40 \mathrm{dBsm}$ and decreases the ACS by about $60 \mathrm{dBsm}$. The frequency domain predicted distributions and vector fields for the electric field, the power density, current density and the electric and magnetic field energy densities of this optimized active CC-CNP are shown at its resonance frequency in Figure 7. In Figures $7 \mathrm{a}$ and $7 \mathrm{~b}$, the electric field and power flow distributions also have a dipole mode but at much higher levels than the passive ones. The power flow is 

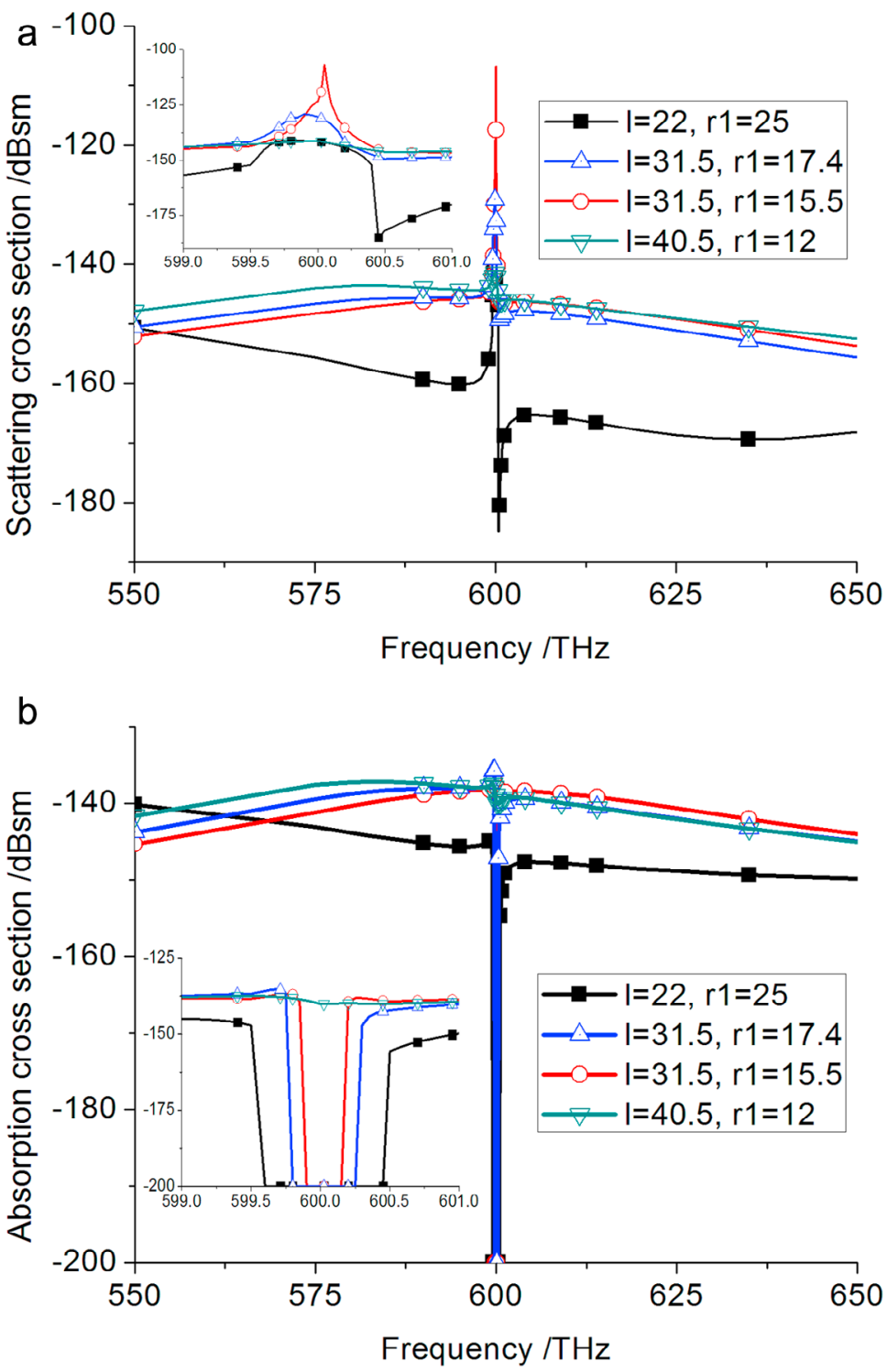

Figure 6. Cross sections, sweeping $r 1: 12.0 \mathrm{~nm} \rightarrow 25.0 \mathrm{~nm}$, and $l: 22.0 \mathrm{~nm} \rightarrow 40.5 \mathrm{~nm}$. (a) Scattering and (b) absorption cross section values for the indicated cases.

clearly outwards, away from the center of the gain region. The corresponding current distribution is shown in Figure 7c; it also exhibits the dipole mode. Moreover, it is quite clear that now there are very large currents flowing through the gain region rather than mainly in the shell, as it is for nonresonant frequencies or in the passive case. Note that as indicated by equation (1), the relative permittivity of silver includes both real and imaginary components. Similarly, from equations (2)-(4) and Geng et al. [2011b, Figure 1], the permittivity of the gain medium also has real and imaginary parts. Thus, the current density in both the silver shell and the core of the CC-CNP will include contributions from conduction and displacement currents. As shown in Figure $4 c$, the currents in the shell dominate when the core is passive. These are conduction currents arising from the large imaginary part of the permittivity. On the other hand, those in the passive core are dominated by the much smaller displacement currents which are associated with the real part of the permittivity. In contrast, when the gain becomes large as it does at the resonance frequency, the effective conductivity of the gain medium will become large. Therefore, if the local electric field is also large, there will be very large conduction currents flowing in the gain medium. Because the gain coremetal shell configuration is selected purposely to have a juxtaposition of media with opposite signs of the real part of the permittivity to achieve an electrically small resonator [Engheta and Ziolkowski, 2006], very large electric fields occur in the core [Gordon and Ziolkowski, 2007a; Arslanagić and Ziolkowski, 2010; Gordon and Ziolkowski, 2007b; Geng et al., 2011a]. Consequently, the CC-CNP configuration will produce very large currents in the core at the resonance frequency as illustrated in Figure 7c. Moreover, notice that the current density in the gain medium is oriented opposite to that in the silver shell. This behavior is expected because the imaginary parts of the permittivity in the core and in the shell have opposite signs, i.e., the sign of 

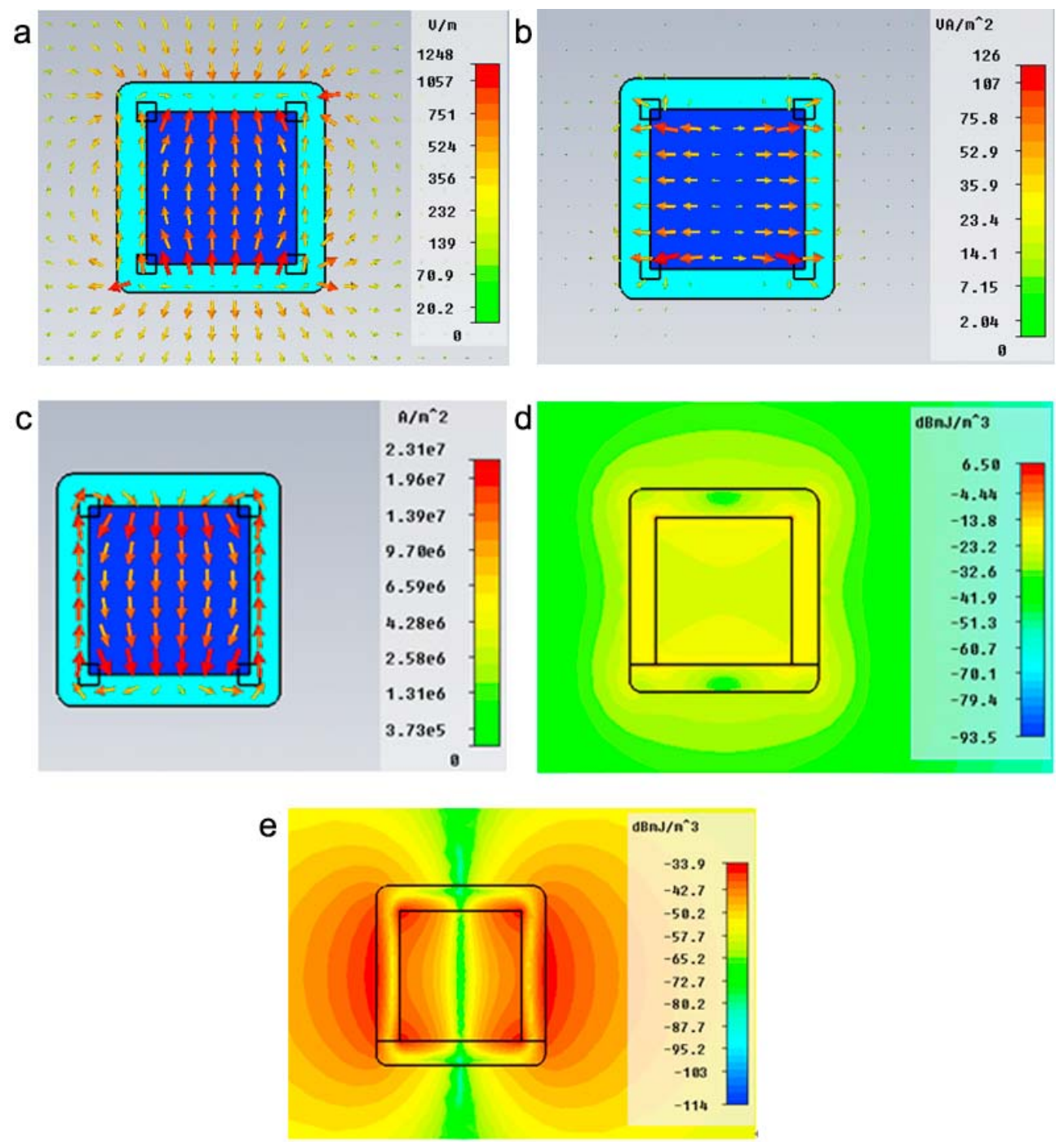

Figure 7. Predicted distributions and vector fields in the y-plane for the active CC-CNP at its resonance frequency, $f_{\text {res }}=600.05 \mathrm{THz}$. (a) E field, (b) power density, (c) current density, (d) E-field energy density, and (e) H-field energy density.

the imaginary part of permittivity of the gain medium around the resonance frequency is negative while it is positive in the silver shell. These enhanced current distributions produce the observed enhanced radiated power levels. The far field patterns are given in Figure 8; again a very strong dipole mode is formed at the resonance frequency. The E-field and the H-field energy densities shown, respectively, in Figures $7 \mathrm{~d}$ and $7 \mathrm{e}$ are very high near the corners of the gain region, but their distributions are clearly dominated by the dipole mode.

\section{Active CC-CNP Numerical Results: EHD Excitation}

[20] The CC-CNP dimensions for an EHD excitation remain the same as those obtained above for the maximizedresponse plane wave case: $l=31.5 \mathrm{~nm}, r 1=15.5 \mathrm{~nm}$ and $d d=$ $6.0 \mathrm{~nm}$. The shell remains silver; the cavity remains filled with the same gain material. The EHD is realized as a small $\mathrm{z}$-directed current element located between the points ( $r r a, 0$, $z c-1 \mathrm{~nm})$ and $(\mathrm{rra}, 0, z c+1 \mathrm{~nm})$. Thus, it is parallel to the axis of the CC-CNP. It is assumed to have the current dipole moment: $I_{0} d=2 \times 10^{-12}$ A-m.

[21] To understand the radiation characteristics of the EHD in the presence of the active CC-CNP, we considered several parameter studies of this nano-antenna system. First, we considered scanning the EHD position along the $x$ axis, $r r a: 0 \rightarrow 45.0 \mathrm{~nm}$, for the fixed $\mathrm{z}$ axis location: $z c=1 / 2$, i.e., along the horizontal centerline of the CC-CNP. The power ratios and $\mathrm{ACSs}$ for the points $\mathrm{A}, \mathrm{B}, \mathrm{C}, \mathrm{D}$ and $\mathrm{G}$, indicated in Figure 9, are given in Figure 10. Similar to the corresponding results in Figure 6, there are strong peaks in the power ratios at the resonant frequency in Figure 10a. The maximum of these peaks, $66.298 \mathrm{~dB}$, is found to occur when the EHD is located at point A $(r r a=45.0 \mathrm{~nm}, z c=1 / 2)$. Similarly, the deepest minimum in Figure $10 \mathrm{~b}$ occurs for this EHD location. This off-center result is very similar to the two-dimensional case results (infinite line source exciting a passive coated infinite cylinder) [Arslanagić et al., 2006]. The dipole mode can only be excited if the line source is 

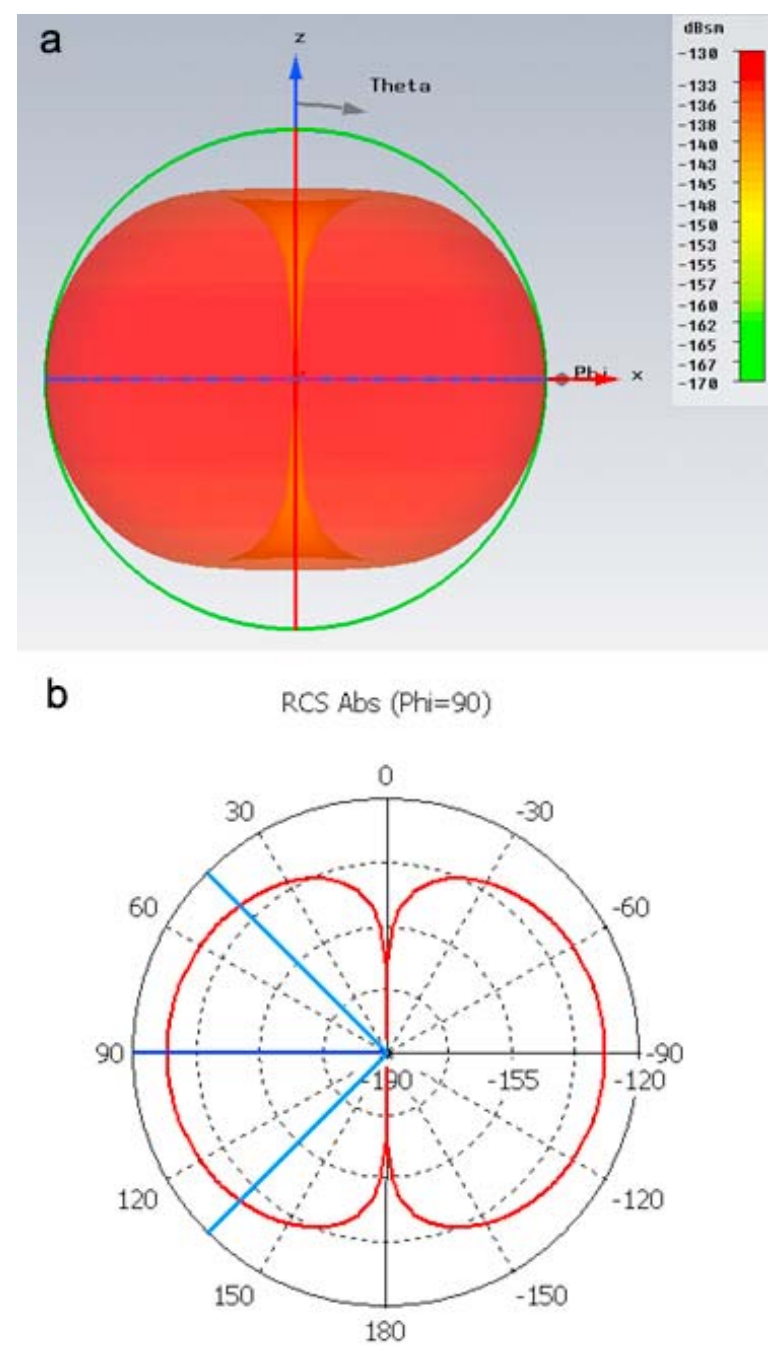

Theta / Degree vs, dBsm

Figure 8. The far-field behavior at the $600.05 \mathrm{THz}$ resonance frequency of the active CC-CNP; (a) 3D radar crosssection (RCS) and (b) 2D cut of the RCS in the $\phi=0^{\circ}$ plane.

offset and it is the dipole mode that produces the largest enhancements. In this three-dimensional case, the finiteness of the structure impacts these results. Because the corners generate all modes once excited, they provide the coupling to the dipole mode even when the EHD is symmetrically located at the center. However, because the length is about twice the radius, the CC-CNP looks a bit like the infinite cylinder case in its horizontal center plane. Thus, the offset point $A$, rather than the center point $G$, produces the largest power ratio value. The closest proximity of the largest fields from the EHD to the plasmon-gain region interface leads to the largest responses [Arslanagic et al., 2006]. Once the EHD is located on the outside of the CC-CNP, the peak power decreases as the EHD location is moved further away from it, i.e., with increasing $r r a$. Similarly, the maximum depth of the ACS null decreases with increasing rra once the EHD is outside the CC-CNP. The field distributions and vector fields when the EHD is located at the offset point A and is excited at the resonance frequency, $599.972 \mathrm{THz}$, are illustrated in Figure 11. The E field, power density and E-field and $\mathrm{H}$-field energy densities are all symmetrical and have a dipole mode form. The bulk currents at that resonance frequency clearly flow in the gain material, as well as in the silver shell. They again have the form which produces the dipole mode and a large enhancement of the total radiated power.

[22] We next considered scanning the EHD position along the vertical centerline of the active CC-CNP, i.e., along $\mathrm{z}$ axis where $r r a=0$. The power ratios and ACSs at the points $\mathrm{E}, \mathrm{F}, \mathrm{O}, \mathrm{H}$ and $\mathrm{G}$, indicated in Figure 12, are given in Figure 13. The maximum of these peaks, $52.075 \mathrm{~dB}$, is found to occur when the EHD is located at the center, at point $\mathrm{G}(r r a=0 \mathrm{~nm}, z c=1 / 2)$, at the resonance frequency: $599.972 \mathrm{THz}$. The field from the EHD has its maximum coupling to the corners and, hence, to exciting the dipole mode. Similarly, the deepest minimum in Figure 13b occurs for this EHD location. Once the EHD is located on the outside of the CC-CNP, the peak power decreases significantly as the EHD location is moved further away from it along the $\mathrm{z}$ axis. The decrease is faster than in the horizontal scan because the EHD produces no field along the $\mathrm{z}$ axis. Once it moves away from the CC-CNP, it thus couples poorly to it. The maximum depth of the null increases as the EHD moves to the center of the active CC-CNP. The farfield pattern when the EHD is located at point $\mathrm{G}$ is shown in Figure 14. It has the expected dipole behavior. The maximum directivity is $1.876 \mathrm{dBi}$.

\section{Active CC-CNP Array: EHD Excitation}

[23] From the above analyses, the active CC-CNP produces significant enhancements of the total radiated power when excited by an EHD. To understand if there are possible means to achieve directivity enhancements associated with such a nano-antenna, we considered the behavior of a $2 \times 2$ array of these CC-CNPs. This is the three-dimensional nano-analogue of the line source excited, array of metamaterialbased infinite-cylinders studied previously in the microwave regime [Arslanagić and Breinbjerg, 2011]. The array was first excited by an EHD located at its geometric center as shown in Figure 15. The size of each silver coated, gain impregnated silica core, CC-CNP remained at $l=31.5 \mathrm{~nm}$, $r 1=15.5 \mathrm{~nm}$, and $d d=6.0 \mathrm{~nm}$. The size of the array was

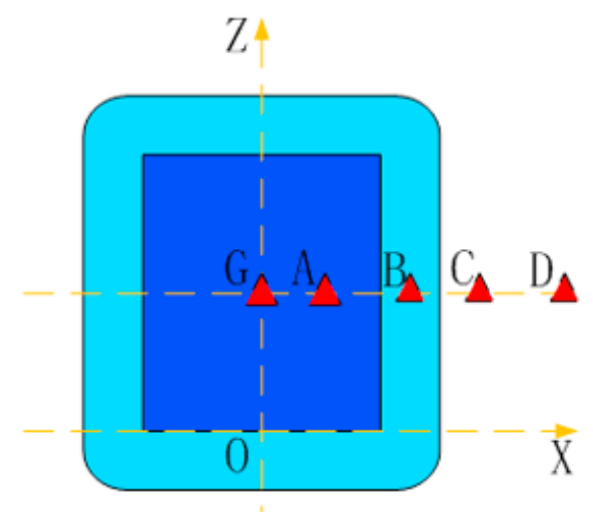

Figure 9. Scanning the EHD locations along the horizontal centerline of the active CC-CNP. 

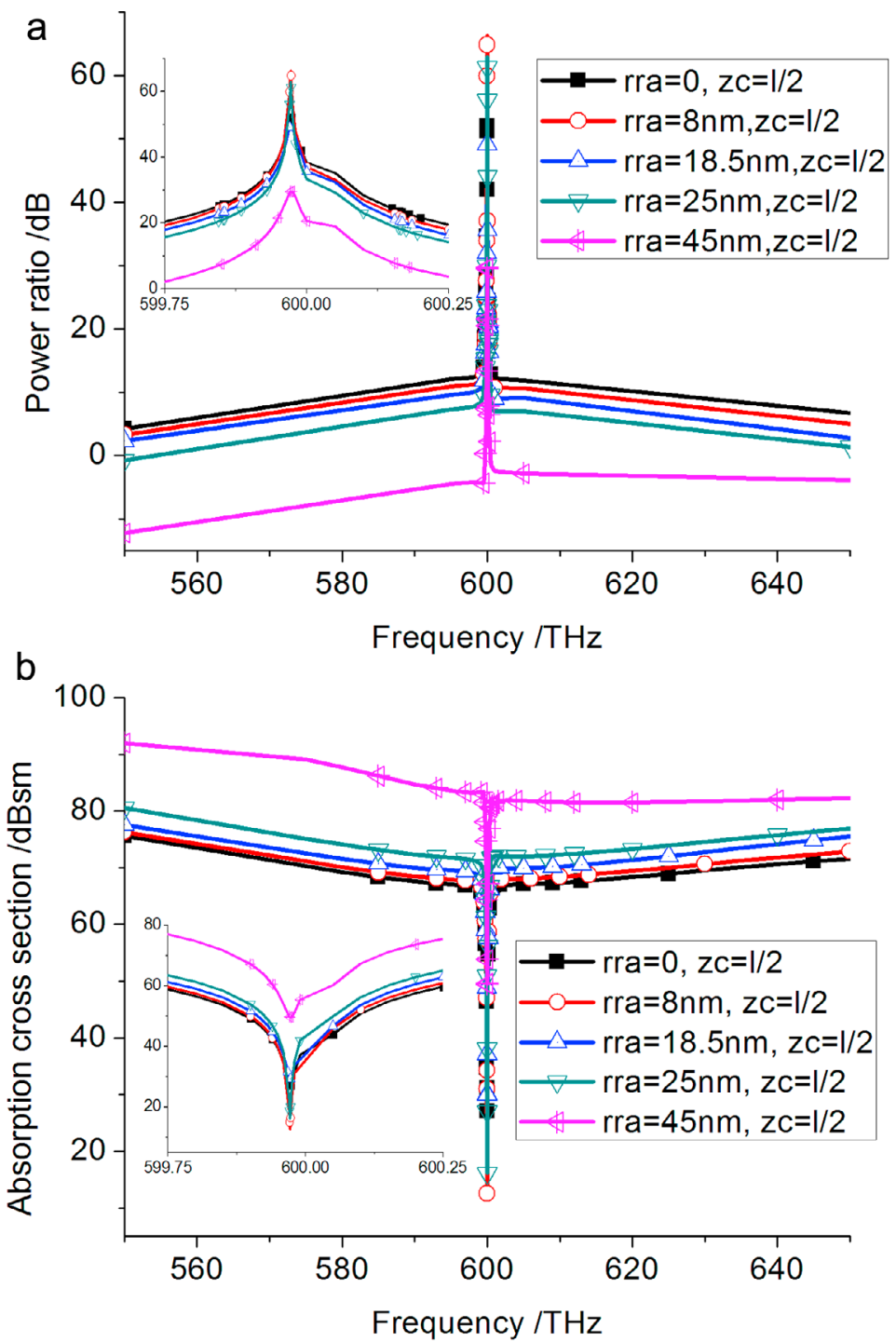

Figure 10. Results for the different EHD locations along the horizontal centerline of the active CC-CNP:

(a) power ratio and (b) absorption cross section.

taken to be the radius of the circle on which the centers of the four CC-CNPs reside, and is labeled rra. The EHD is again oriented along the $\mathrm{z}$ axis, parallel to the long axis of all of the CC-CNPs, and has the current dipole moment $I_{0} d=2 \times 10^{-12} \mathrm{~A}-\mathrm{m}$.

[24] We first scanned the radius rra to understand how the coupling between the individual CC-CNPs would affect the power ratios and ACSs. These curves are shown in Figure 16. It is clear that there is a large peak in the power ratio curves, as well as a corresponding depression in the ASC curves at frequencies close to $600 \mathrm{THz}$, where the individual CC-CNPs are resonant. The actual maximum of the power ratio peak is $10.52 \mathrm{~dB}$ at the resonance frequency $f_{\text {res }}=599.92 \mathrm{THz}$ for $r r a=260.0 \mathrm{~nm}$. The minimum of the ASC, $75.23 \mathrm{dBsm}$, occurs at the same frequency for the same configuration. We note that this value for $r r a$ is slightly larger than $\lambda_{0} / 2$, the resonance wavelength being $\lambda_{0}=$ $499.72 \mathrm{~nm}$. Consequently, this configuration is essentially an antenna array with four independent (uncoupled) elements. This behavior is illustrated, for example, with the electric field distribution and the H-field energy distribution near the array given in Figure 17 at the resonance frequency, $599.92 \mathrm{THz}$. One can see that the EHD excites independent dipole modes in each CC-CNP. The far field patterns generated by this array are shown in Figure 18. Because the EHD excitation is common to each CC-CNP, they are excited in-phase. This causes the four symmetrical lobes in the H-plane pattern seen in Figure 18b. The maximum directivity is $8.41 \mathrm{~dB}$ in the H-plane (xy-plane) at $599.92 \mathrm{THz}$. This is a $6.524 \mathrm{dBi}$ increase over a single element. Adding more CC-CNPs, even higher directivity beams would be possible. Note that not only enhancements are possible, but the array can substantially reduce the total radiated power as shown in Figure 16a for the $r r a=150 \mathrm{~nm}$ case. This is analogous to the "active cloaking" discussed by Arslanagic and Ziolkowski [2010] or could be considered as active jamming of the EHD. The EHD excites the active CC-CNPs into their resonant states. The latter then emit a strong signal 

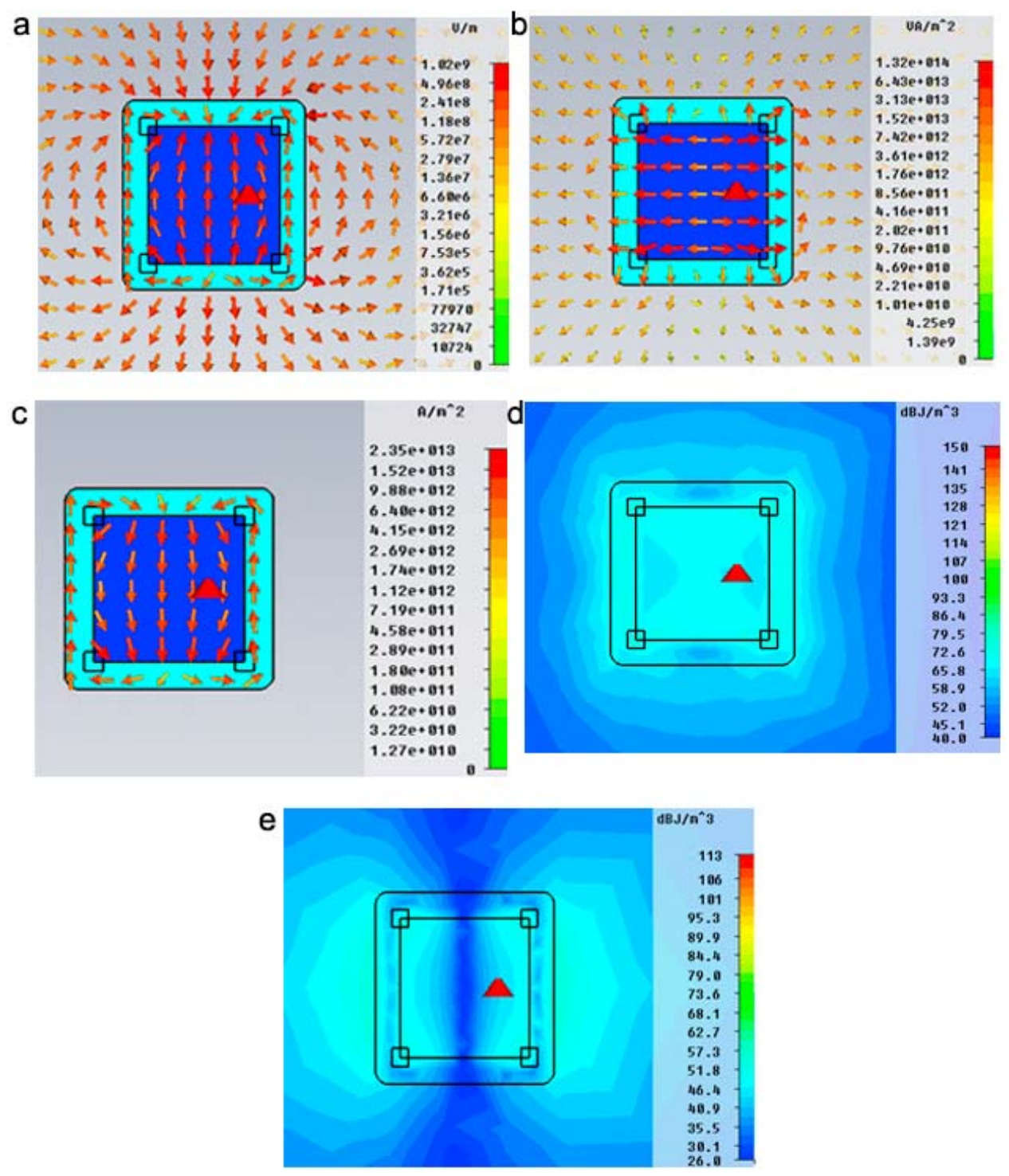

Figure 11. Field distributions and vector fields at the resonance frequency $f_{\text {res }}=599.972 \mathrm{THz}$, when the EHD is located at point A within the active CC-CNP. (a) E field, (b) power flow, (c) current flow, (d) E-field energy density, and (e) H-field energy density.

that actively "jams" the output signal of the EHD. The radiated power ratio falls to $-12.429 \mathrm{~dB}$, a $8.771 \mathrm{~dB}$ and a $5.938 \mathrm{~dB}$ difference, respectively, from the maximum and background values. This is significantly $(14.305 \mathrm{~dB})$ smaller than the dipole value itself.

[25] On the other hand, with $r r a=45.0 \mathrm{~nm}$, the elements are separated by a distance slightly more than $\lambda_{0} / 10$ and, hence, are tightly coupled to each other and to the EHD. The impact of this coupling behavior was also discussed in the analogous microwave problem [Engheta and Ziolkowski, 2006]. The EHD and the CC-CNPs act as a composite unit rather than five separate ones. This behavior is observed from the near field distributions shown in Figure 19. The electric field, Figure 19a, shows the intense coupling between the EHD and the CC-CNPs; the power density, Figure 19b shows the net outflow of power from the composite unit. This composite response is further emphasized by the far field patterns of this array at its resonance

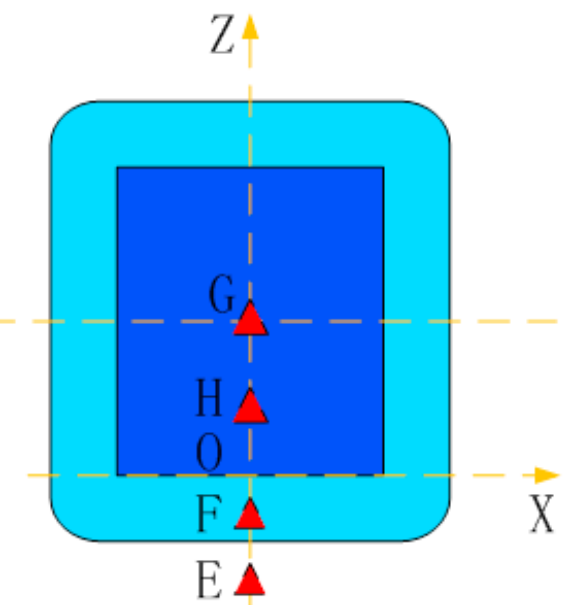

Figure 12. Scanning the EHD locations along the vertical centerline of the active CC-CNP. 

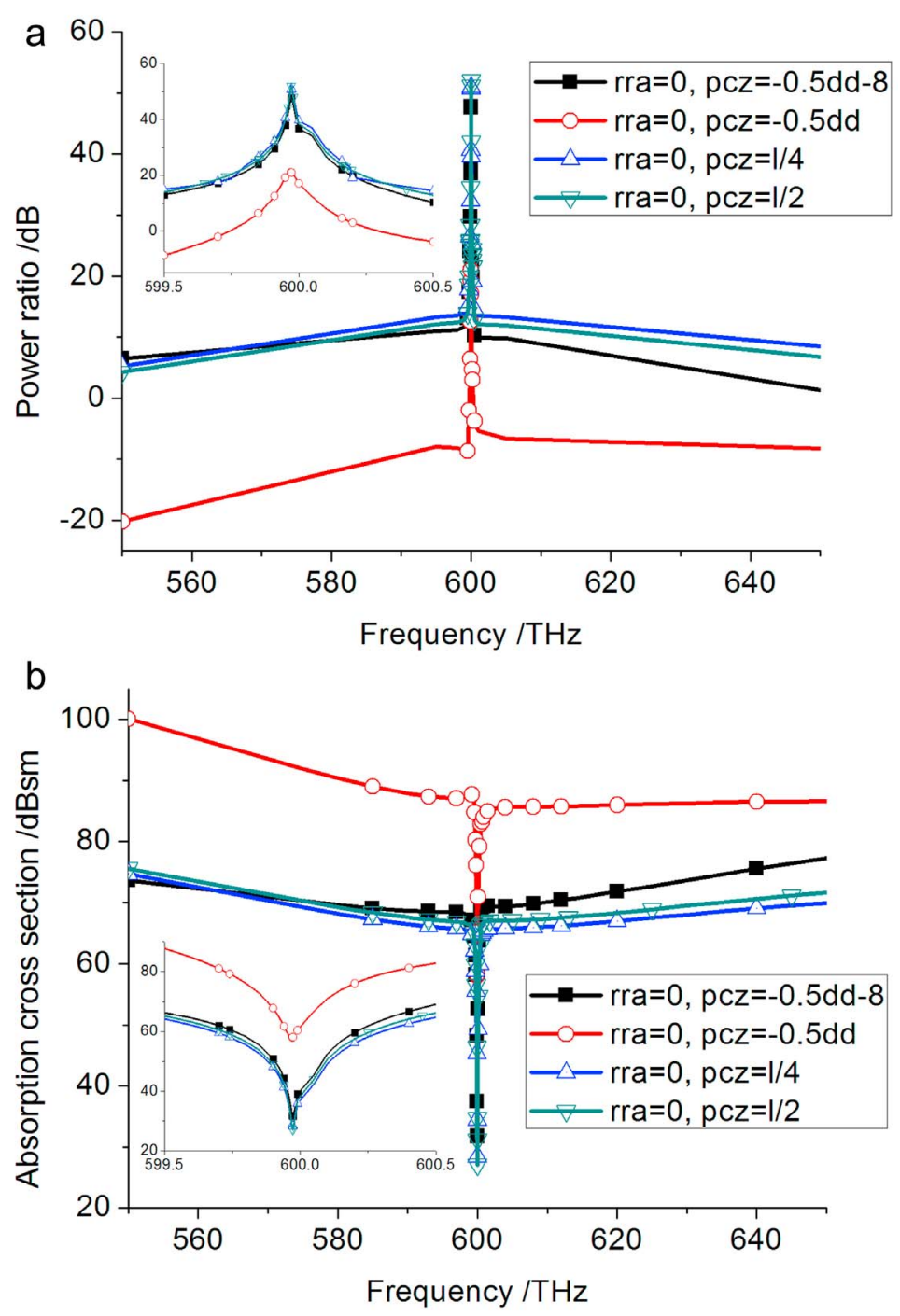

Figure 13. Results for the different EHD locations along the vertical centerline of the active CC-CNP: (a) power ratio and (b) absorption cross section. 


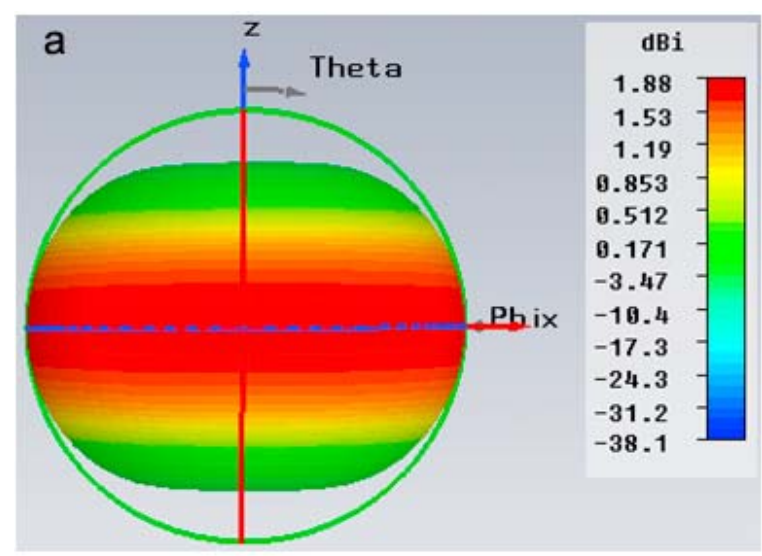

b

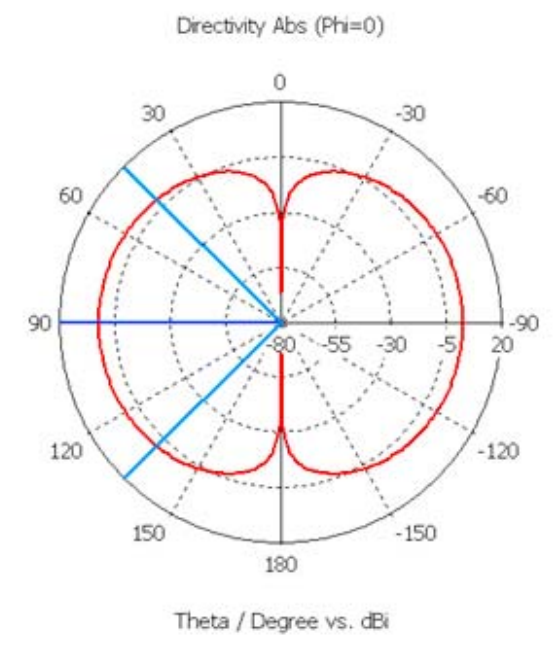

Figure 14. The far-field behavior at the $599.972 \mathrm{THz}$ resonance frequency of the active CC-CNP, which is driven by EHD at point G; (a) 3D radar cross-section (RCS) and (b) 2D cut of the RCS in the $\phi=0^{\circ}$ plane.

frequency, $599.92 \mathrm{THz}$, are shown in Figure 20. The H-plane is almost omni-directional and the E-plane exhibits a dipole pattern.

[26] Finally, we considered the $2 \times 2$ array with the $r r a=$ $260.0 \mathrm{~nm}$ separation and the EHD displaced $50 \mathrm{~nm}$ along the $+x$ axis. The power ratios and the ASCs for the centered and off-center ("biased") EHD cases are compared in Figure 21. The peak of the power ratio is $15.959 \mathrm{~dB}$ higher in the offcenter EHD case, but blue-shifted to a slightly higher frequency, $\mathrm{f}=599.94 \mathrm{THz}$. The depression of the ASC for the offset EHD case is correspondingly lower. The electric field and H-field energy distributions are shown in Figure 22. Only slight variations from the centered case are observed for this offset case. On the other hand, the far-field patterns and the associated directivities are impacted more as shown in Figure 23. In a plane perpendicular to the plane of the array, the dipole pattern has been modified and the presence of a higher order mode is obvious. In the plane of the array, the pattern is rather asymmetric; its peaks are larger now toward the direction of the offset. Moreover, note that there is slight decrease in the peak value because all four elements of the array are no longer completely in phase because of the offset.

\section{Conclusions}

[27] An investigation of the electromagnetic behavior of passive and active, closed, cylindrical, coated nano-particles were presented. Both frequency and time domain results for plane wave excitation of passive CC-CNPs were considered to verify the frequency solver predictions. The properties of the active CC-CNPs were then studied. It was demonstrated that whether the active CC-CNPs were excited by plane wave or EHD fields, they could be designed to be resonant and their fundamental radiated field was a dipole mode. The scattering cross section for a specific plane wave case was increased by $40 \mathrm{dBsm}$ at resonance; the maximum of the power ratio for the corresponding EHD excitation was increased by $66.3 \mathrm{~dB}$. Various positions of the driven EHD were considered to characterize the power ratio enhancements of the resulting nano-antennas. A $2 \times 2$ array of active CC-CNPs was considered. Enhanced directivity was demonstrated when the CC-CNPs in the array were essentially uncoupled from one another and were excited in-phase by a centrally located EHD. When the CC-CNPs were tightly coupled, they acted together as a composite nano-antenna. For an in-between array size, the CC-CNPs acted together to jam the EHD signal, effectively cloaking it to a far-field observer. An offset EHD excitation of the array showed that the far-field patterns could be controlled by the EHD location. The polarization of the excitation fields were taken to be along the major axis of the CC-CNP throughout the
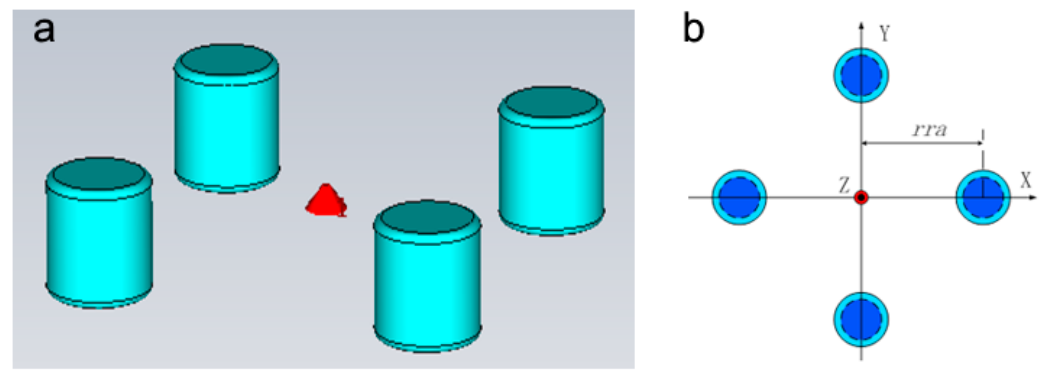

Figure 15. Geometry of the $2 \times 2$ CC-CNP array excited by an EHD at its center: (a) perspective view and (b) top view. 

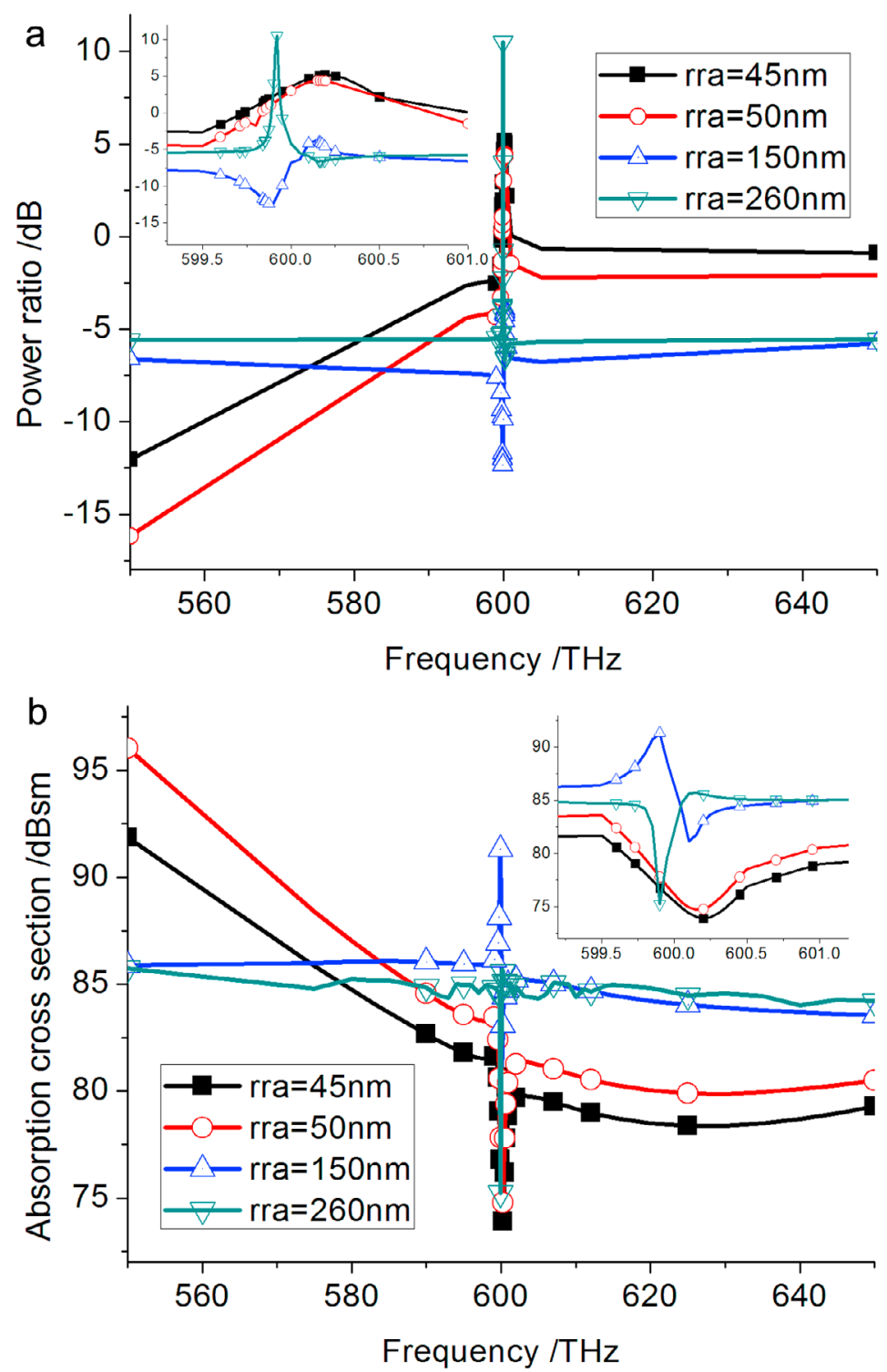

Figure 16. Results for the $2 \times 2$ CC-CNP array excited by an EHD at its center for a scan of the array radius $r r a: 45.0 \mathrm{~nm} \rightarrow 260.0 \mathrm{~nm}$ : (a) power ratios and (b) absorption cross sections. 


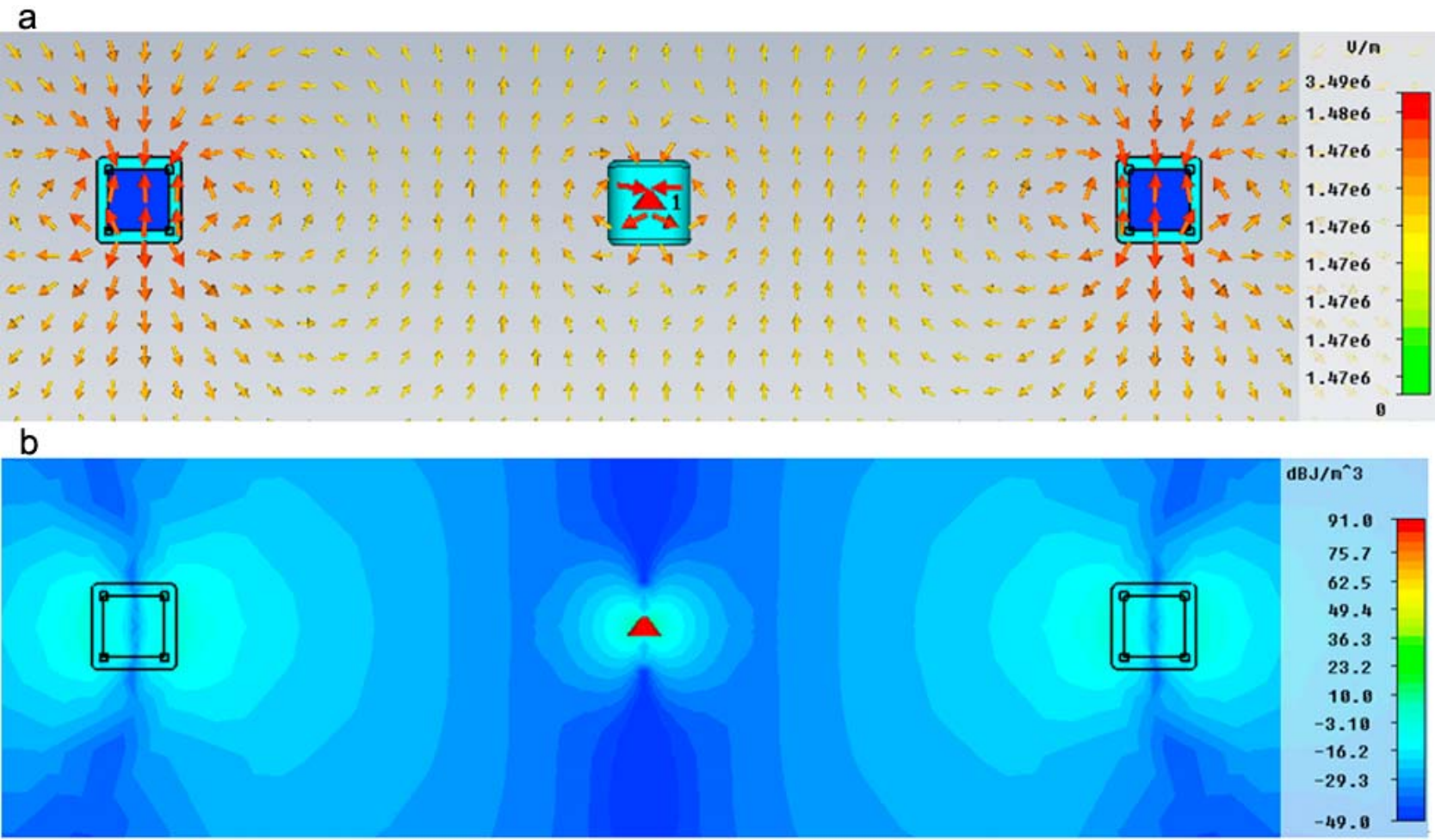

Figure 17. Results for the $2 \times 2$ array of active CC-CNPs that are separated by $r r a=260.0 \mathrm{~nm}$ from the driven EHD at the array center at the resonance frequency $f_{\text {res }}=599.92 \mathrm{THz}$ : (a) electric field and (b) H-field energy distributions. 

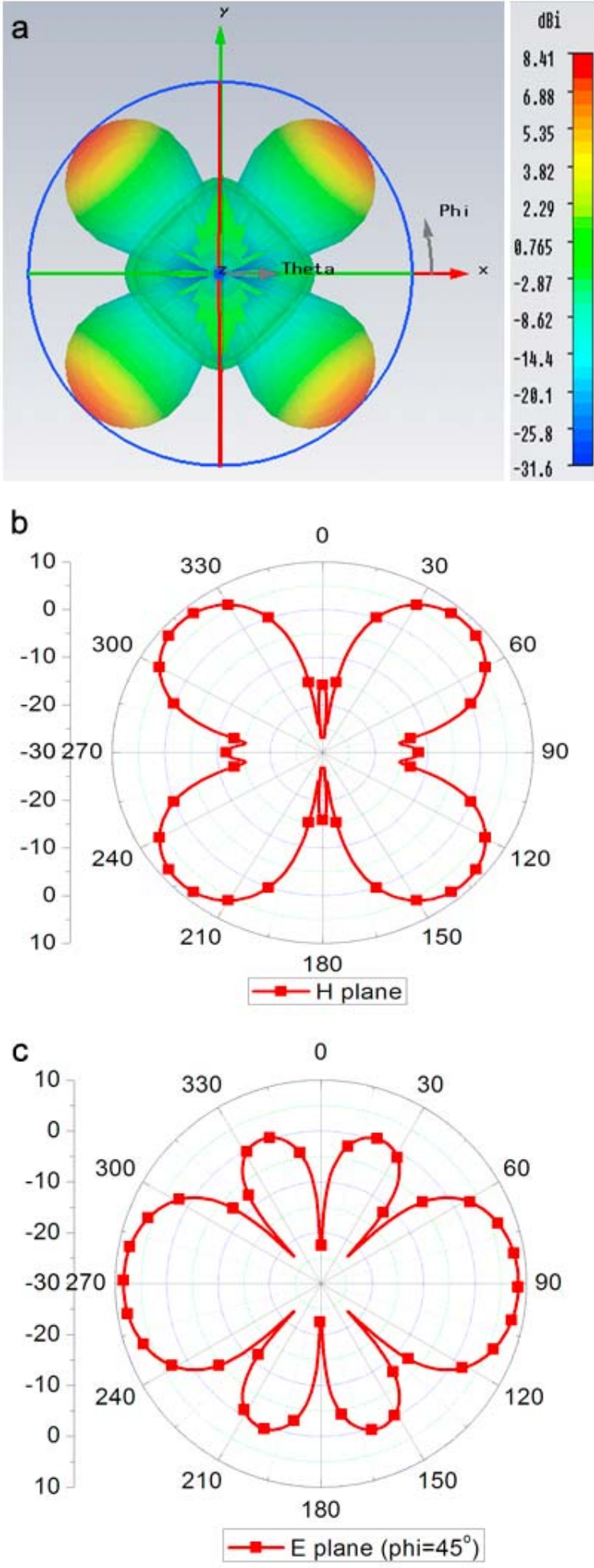

Figure 18. Far field patterns of the $2 \times 2$ array of CC-CNPs with $r r a=260.0 \mathrm{~nm}$ excited by the EHD at its center at the resonance frequency $f_{\text {res }}=599.92 \mathrm{THz}$ : (a) 3D far field pattern, (b) E-plane pattern for $\phi=45^{\circ}$, and (c) H-plane pattern. 
a

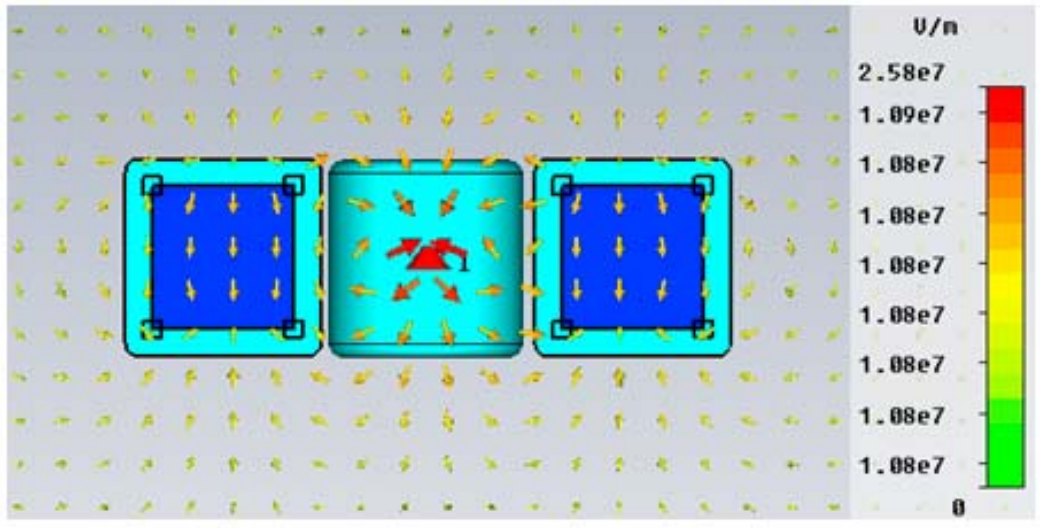

b

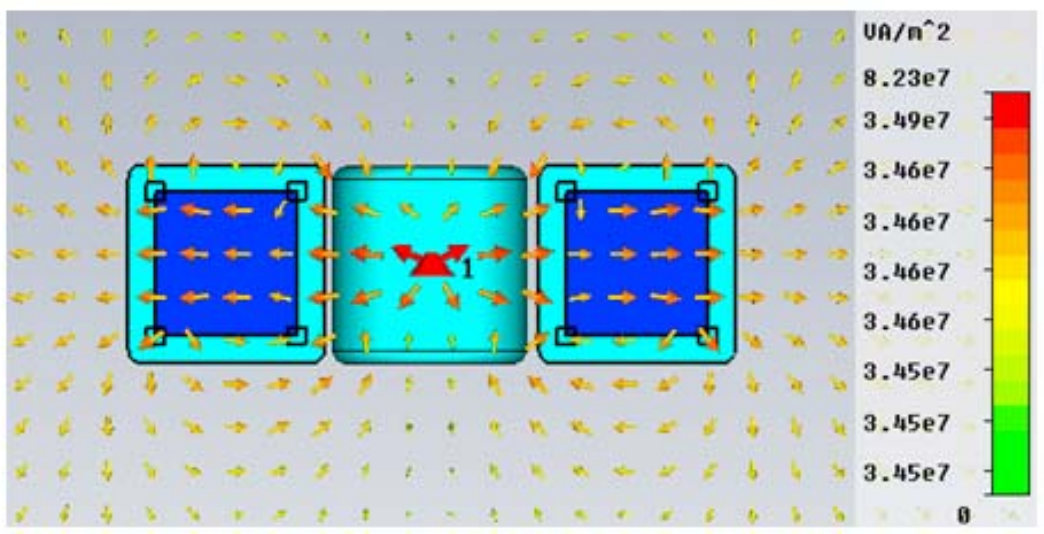

Figure 19. Results for the $2 \times 2$ array of active CC-CNPs that are separated by $r r a=45.0 \mathrm{~nm}$ from the driven EHD at the array center at the resonance frequency $f_{\text {res }}=599.92 \mathrm{THz}$ : (a) electric field and (b) power density distributions. 

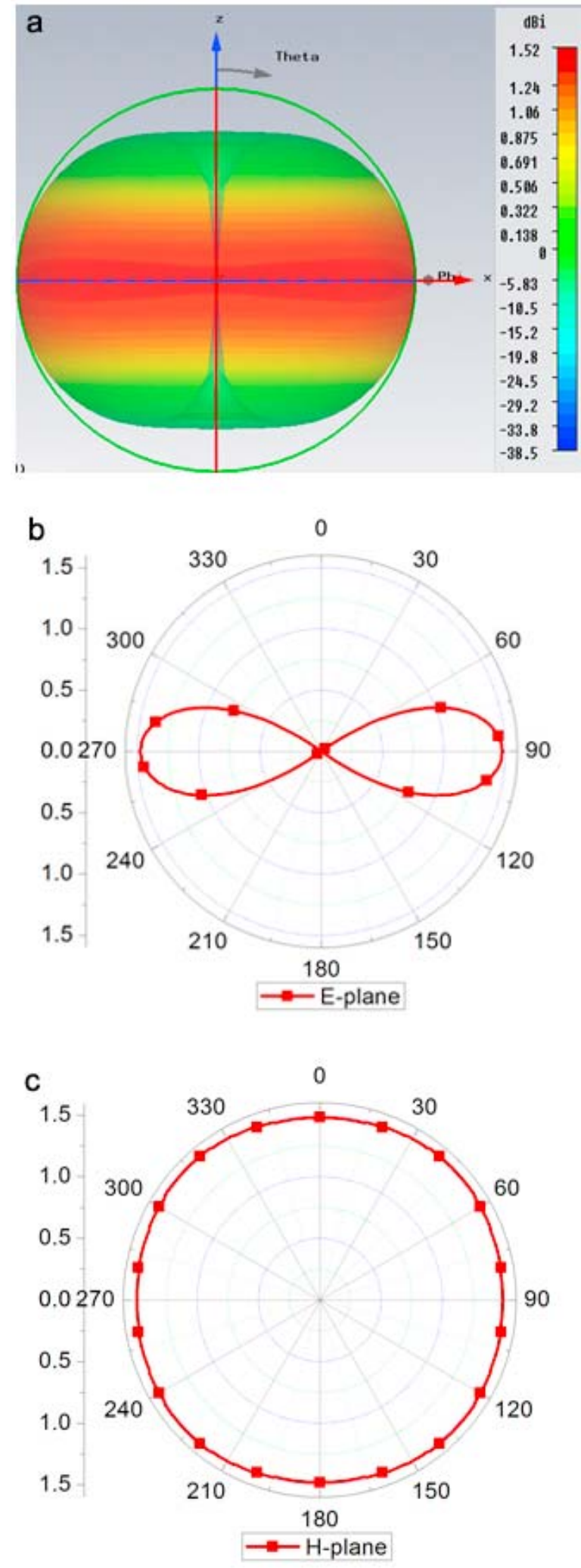

Figure 20. Far field patterns of the $2 \times 2$ array of CC-CNPs with $r r a=45.0 \mathrm{~nm}$ excited by the EHD at its center at the resonance frequency $f_{\text {res }}=599.92 \mathrm{THz}$ : (a) 3D pattern, (b) E-plane pattern for $\phi=0^{\circ}$, and (c) H-plane pattern. 

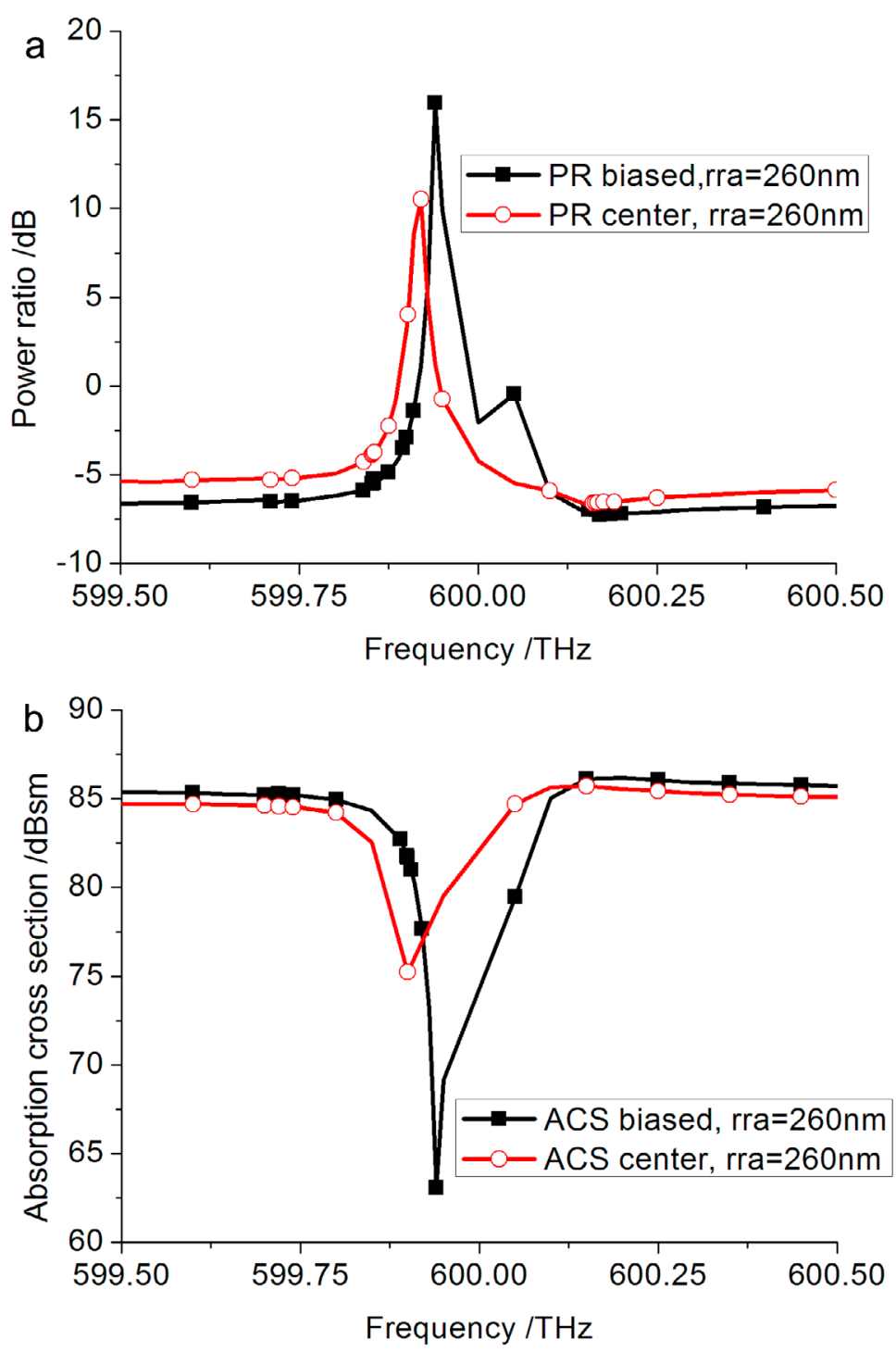

Figure 21. Comparison of the EHD centered and off-set $2 \times 2$ CC-CNP arrays. The EHD is offset $50.0 \mathrm{~nm}$ along the $+x$ axis: (a) power ratios and (b) absorption cross sections. 


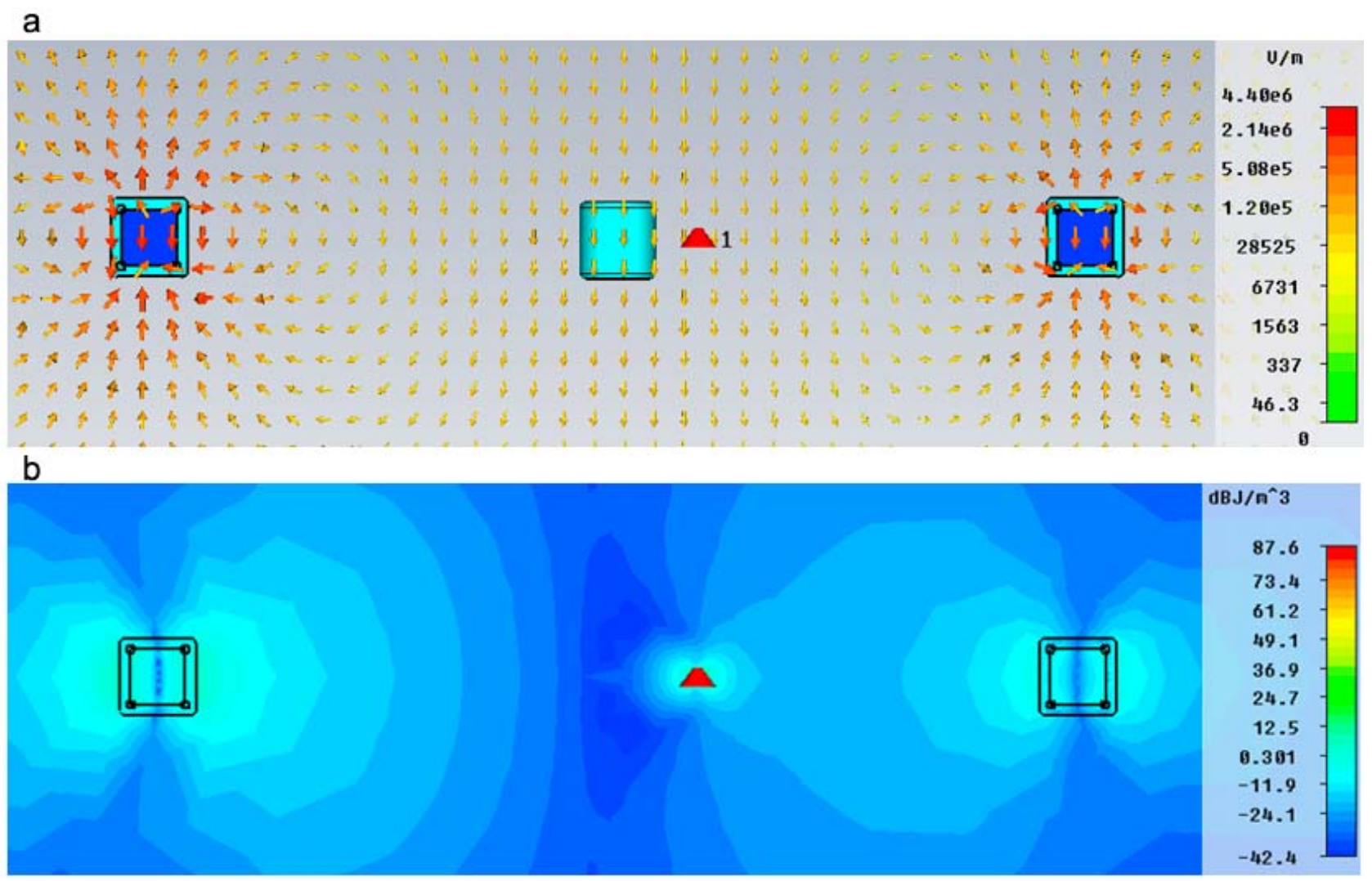

Figure 22. Results for the $2 \times 2$ array of active CC-CNPs that are separated by $r r a=260.0 \mathrm{~nm}$ from the center of the array. The EHD is offset along the $x$ axis by $50.0 \mathrm{~nm}$ and is driven at the resonance frequency $f_{\text {res }}=599.94 \mathrm{THz}$ : (a) electric field and (b) H-field energy density distributions. 

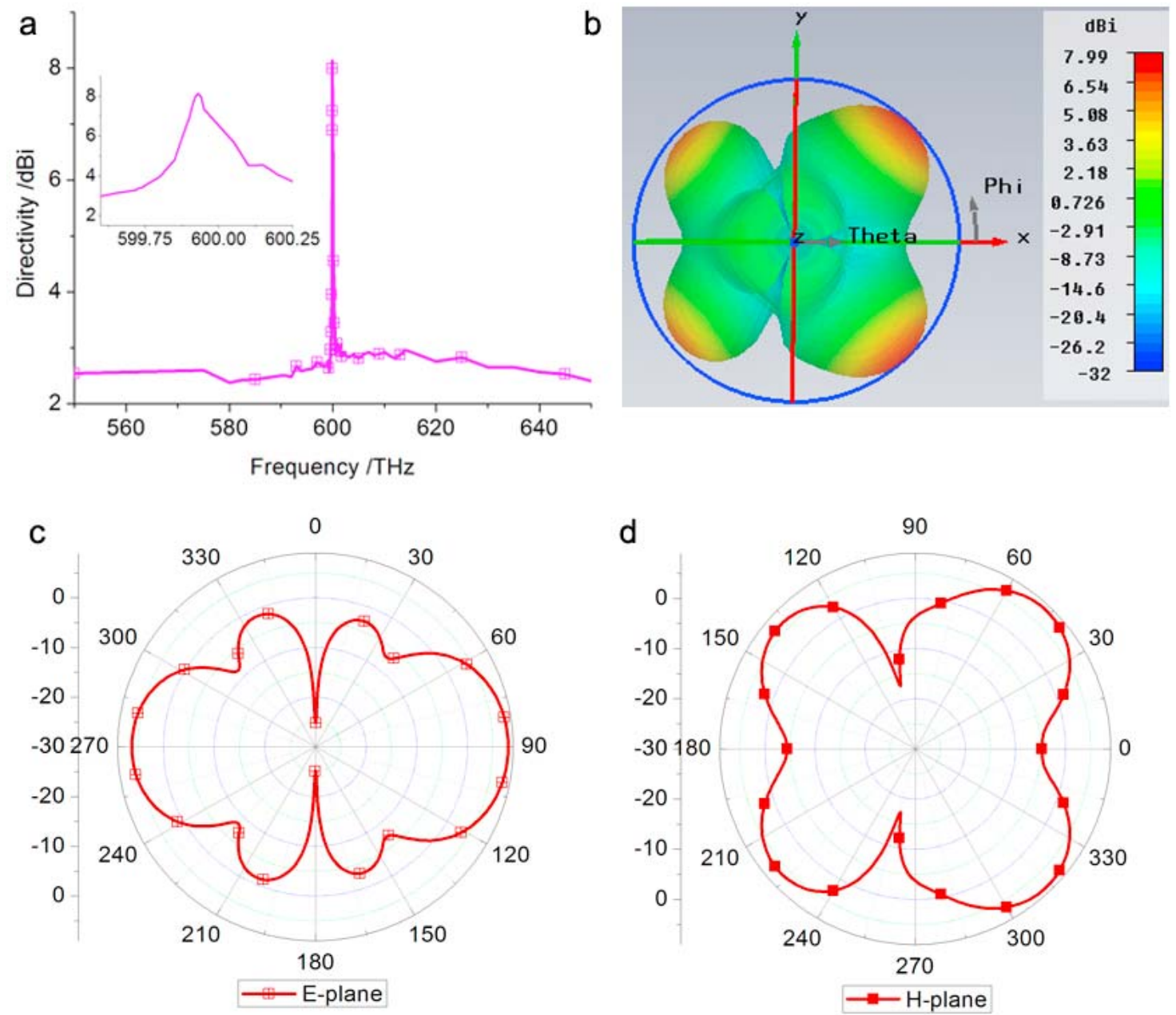

Figure 23. Far-field behavior of the $2 \times 2$ array of active CC-CNPs with $r r a=260.0 \mathrm{~nm}$ excited by the $50.0 \mathrm{~nm}$ offset EHD at the resonance frequency $f_{\text {res }}=599.94 \mathrm{THz}$. (a) Directivity, (b) 3D pattern, (c) E-plane pattern for $\phi=47^{\circ}$, and (d) H-plane pattern.

reported studies. Currently, other polarization behaviors are being explored. The outcome of these investigations will be reported elsewhere.

[28] Acknowledgments. This work was supported in part by '973' (2009CB320403); the Natural Science Foundation of Shanghai (10ZR1416600); the Doctoral Fund of Ministry of Education of China (20090073120033); the National Science and Technology of major projects (2011ZX03001-007-03); the National Science Fund for Creative Research Groups (60821062); Scientific Research Foundation for Returned Scholars, Ministry of Education of China; and by ONR Contract number H940030920902. The authors would like to thank Sawyer Campbell, College of Optical Sciences, University of Arizona, for several enlightening technical discussions.

\section{References}

Aden, A. L., and M. Kerker (1951), Scattering of electromagnetic waves from two concentric spheres, J. Appl. Phys., 22, 1242-1246, doi:10.1063/ 1.1699834.

Arslanagić, S., and O. Breinbjerg (2011), Sub-wavelength resonances in metamaterial-based multi-cylinder configurations, Materials, 4, 117-130, doi:10.3390/ma4010117.

Arslanagić, S., and R. W. Ziolkowski (2010), Active coated nano-particle excited by an arbitrarily located electric Hertzian dipole-resonance and transparency effects, J. Opt., 12, 024014.

Arslanagić, S., R. W. Ziolkowski, and O. Breinbjerg (2006), Excitation of an electrically small metamaterial-coated cylinder by an arbitrarily located line source, Microwave Opt. Technol. Lett., 48, 2598-2606, doi:10.1002/mop. 21990 .

Arslanagić, S., R. W. Ziolkowski, and O. Breinbjerg (2007), Radiation properties of an electric Hertzian dipole located near-by concentric metamaterial spheres, Radio Sci., 42, RS6S16, doi:10.1029/2007RS003663.

Atwater, H. A., and A. Polman (2010), Plasmonics for improved photovoltaic devices, Nat. Mater., 9, 205-213, doi:10.1038/nmat2629.

Balanis, C. A. (2005), Antenna Theory: Analysis and Design, 3rd ed., John Wiley, Hoboken, N. J.

Engheta, N., and R. W. Ziolkowski (Eds.) (2006), Metamaterials: Physics and Engineering Explorations, John Wiley, Hoboken, N. J.

Erentok, A., O. S. Kim, and S. Arslanagić (2009), Cylindrical metamaterialbased sub-wavelength antenna, Microwave Opt. Technol. Lett., 51, 1496-1500, doi:10.1002/mop. 24386.

Ferry, V. E., L. A. Sweatlock, D. Pacifici, and H. A. Atwater (2008), Plasmonic nanostructure design for efficient light coupling into solar cells, Nano Lett., 8, 4391-4397, doi:10.1021/n18022548.

Geng, J., R. W. Ziolkowski, R. Jin, and X. Liang (2011a), Numerical study of active open cylindrical coated nano-particle antennas, IEEE Photonics J., 3, 1093-1110, doi:10.1109/JPHOT.2011.2173759.

Geng, J., R. W. Ziolkowski, S. Campbell, R. Jin, and X. Liang (2011b), Studies of nanometer antennas incorporating gain material using CST, in 2011 IEEE International Symposium on Antennas and Propagation (APSURSI), pp. 1624-1627, IEEE Press, Piscataway, N. J., doi:10.1109/ APS.2011.5996613.

Gordon, J. A., and R. W. Ziolkowski (2007a), The design and simulated performance of a coated nano-particle laser, Opt. Express, 15, 2622-2653, doi:10.1364/OE.15.002622. 
Gordon, J. A., and R. W. Ziolkowski (2007b), Investigating functionalized active coated nano-particles for use in nano-sensing applications, Opt. Express, 15, 12,562-12,582, doi:10.1364/OE.15.012562.

Grbic, A., L. Jiang, and R. Merlin (2008), Near-field plates: Subdiffraction focusing with patterned surfaces, Science, 320, 511-513, doi:10.1126/ science. 1154753 .

Halas, N. J. (2010), Plasmonics: An emerging field fostered by Nano Letters, Nano Lett., 10, 3816-3822, doi:10.1021/nl1032342.

Hill, M. T. (2010), Status and prospects for metallic and plasmonic nanolasers, J. Opt. Soc. Am. B, 27, B36-B44, doi:10.1364/JOSAB.27.000B36.

Hirsch, L. R., A. M. Gobin, A. R. Lowery, F. Tam, R. A. Drezek, N. J. Halas, and J. L. West (2006), Metal nanoshells, Ann. Biomed. Eng., 34, 15-22, doi:10.1007/s10439-005-9001-8.

Jain, P. K., X. Huang, I. H. El-Sayed, and M. A. El-Sayed (2008), Noble metals on the nanoscale: Optical and photothermal properties and some applications in imaging, sensing, biology, and medicine, Acc. Chem. Res., 41, 1578-1586, doi:10.1021/ar7002804

Johnson, P. B., and R. W. Christy (1972), Optical constants of the noble metals, Phys. Rev. B, 6, 4370-4379, doi:10.1103/PhysRevB.6.4370.

Kawata, S., Y. Inouye, and P. Verma (2009), Plasmonics for near-field nano-imaging and superlensing, Nat. Photonics, 3, 388-394, doi:10.1038/ nphoton.2009.111.

Khlebtsov, N., B. Khlebtsov, E. Panfilova, V. Khanadeev, O. Bibikova, S. Staroverov, G. Terentyuk, V. Rumyantseva, and A. Ivanov (2011) Novel multifunctional nanocomposites for theranostics, SPIE Newsroom, 22 Sept., doi:10.1117/2.1201109.003832.

Li, J., A. Salandrino, and N. Engheta (2007), Shaping light beams in the nanometer scale: A Yagi-Uda nanoantenna in the optical domain, Phys. Rev. B, 76, 245403, doi:10.1103/PhysRevB.76.245403.

Catchpole, K. R., and A. Polman (2008), Plasmonic solar cells, Opt. Express, 16, 21,793-21,800, doi:10.1364/OE.16.021793.

Li, Z., J. Wang, N. Singh, and S. Lee (2011), Optical and electrical study of core-shell silicon nanowires for solar applications, Opt. Express, 19, A1057-A1066, doi:10.1364/OE.19.0A1057.
Lin, A. W. H., N. A. Lewinski, J. L. West, N. J. Halas, and R. A. Drezek (2005), Optically tunable nanoparticle contrast agents for early cancer detection: Model-based analysis of gold nanoshells, J. Biomed. Opt., 10, 064035, doi:10.1117/1.2141825.

Liu, X., and Q. Huo (2009), A washing-free and amplification-free one-step homogeneous assay for protein detection using gold nanoparticle probes and dynamic light scattering, J. Immunol. Methods, 349, 38-44, doi:10.1016/ j.jim.2009.07.015

Mirin, N. A., T. A. Ali, P. Nordlander, and N. J. Halas (2010), Perforated semishells: Far-field directional control and optical frequency magnetic response, ACS Nano, 4, 2701-2712, doi:10.1021/nn100535m.

Noginov, M. A., G. Zhu, R. Bakker, V. M. Shalaev, E. E. Narimanov, S. Stout, E. Herz, T. Suteewong, and U. Wiesner (2009), Demonstration of a spaser-based nanolaser, Nature, 460, 1110-1112, doi:10.1038/ nature08318.

Pala, R. A., J. White, E. Barnard, J. Liu, and M. L. Brongersma (2009), Design of plasmonic thin-film solar cells with broadband absorption enhancements, Adv. Mater., 21,3504-3509, doi:10.1002/adma.200900331.

Shvets, G., S. Trendafilov, J. B. Pendry, and A. Sarychev (2007), Guiding, focusing, and sensing on the subwavelength scale using metallic wire arrays, Phys. Rev. Lett., 99, 053903, doi:10.1103/PhysRevLett.99.053903.

Stockman, M. I. (2010), The SPASER as a nanoscale quantum generator and ultrafast amplifier, J. Opt., 12, 024004.

Wang, D., T. Yangand, and K. B. Crozier (2011), Optical antennas integrated with concentric ring gratings: Electric field enhancement and directional radiation, Opt. Express, 19, 2148-2157, doi:10.1364/OE.19.002148.

Weiland, T. (1998), Time domain electromagnetic field computation with finite difference methods, Int. J. Numer. Modelling, 9, 259-319.

J. Geng, R. Jin, and X. Liang, Electronic Engineering Department, Shanghai Jiao Tong University, Shanghai 200240, China. (gengjunp@sjtu. edu.cn)

R. W. Ziolkowski, Department of Electrical and Computer Engineering, University of Arizona, 1230 E. Speedway Blvd., Tucson, AZ 85721, USA. 